\title{
Exploring German Liquid Democracy - Online-Partizipation auf der lokalen Ebene
}

\author{
Julia Schwanholz $\cdot$ Lavinia Zinser
}

Online publiziert: 7. Juli 2020

(C) Der/die Autor(en) 2020

Zusammenfassung Befunde über den Zustand repräsentativer Demokratie zeigen teilweise eine Unzufriedenheit mit politischen Institutionen, Akteuren und Prozessen der Entscheidungsfindung. Der Staat reagiert hierauf vermehrt mit Instrumenten direkter Demokratie. Dabei ist das Repertoire durch Angebote zur Online-Partizipation ergänzt und ausgeweitet worden. Im Beitrag wird analysiert, wie OnlinePartizipationsangebote auf der lokalen Ebene konkret ausgestaltet sind. Die zentrale Frage lautet, ob die Angebote zu mehr Online-Partizipation anregen oder nicht. Es werden dafür sechs kommunale Plattformen untersucht und verglichen. Deutlich wird dabei zweierlei: die Angebote lassen sich erstens entlang der eingesetzten Software voneinander unterscheiden und strukturieren. Für die Angebotsseite sind zweitens einige konkrete Aspekte aufzuzeigen, wie Partizipation gesteigert (oder auch gehemmt) werden kann.

\section{Exploring German Liquid Democracy-online-participation at the local level}

\begin{abstract}
The state of representative democracy shows some dissatisfaction with political institutions, actors and decision-making processes. The state is increasingly responding to this with instruments of direct democracy. The existing repertoire is supplemented by offers for online participation. The article analyzes what kind of online participation platforms are offered to the citizens on the local level. Our research question focuses on whether or not the offers encourage online participation. For this purpose, six local platforms are examined and compared. The results show that platforms can be differentiated and structured along the software used. More-
\end{abstract}

Dr. J. Schwanholz $(\bowtie) \cdot$ L. Zinser

Institut für Politikwissenschaft, Georg-August-Universität Göttingen, Platz der Göttinger

Sieben 3, 37073 Göttingen, Deutschland

E-Mail: julia.schwanholz@sowi.uni-goettingen.de 
over, some specific aspects are found on the supply side that can increase or inhibit participation.

\section{Einleitung}

Die digitale Transformation durchdringt die Gesellschaft in allen Sphären. Fraglich ist, ob alte Konzepte der Demokratie - wie Repräsentation, Öffentlichkeit und Souveränität - weiterhin gültig oder nicht vielmehr zu überdenken und neu zu definieren sind (Schaal 2015, S. 297 f.). Dahinter stecken grundsätzliche Fragen nach dem Zustand und der Wahrnehmung der Demokratie. In der Literatur herrscht Uneinigkeit darüber, ob die repräsentative Demokratie in der Krise steckt (van Ham et al. 2017; Zittel 2012; Kersting 2013; Kersting et al. 2008; Merkel 2015a; Merkel und Krause 2015; Kriesi 2013, 2014). Weniger strittig ist, dass Bürger der politischen Elite zunehmend misstrauen und das Prinzip der Repräsentativität infrage stellen (Pähle 2008; Kersting 2008; Pickel 2013). Es werden Legitimitätsdefizite konstatiert (Geissel 2008; Kersting 2013) und eine „Demokratisierung der Demokratie“ gefordert (Offe 2003). Allerdings finden sich auch Befunde, wonach pauschale Legitimitätsverluste als Mythos zu werten sind, weil das Vertrauen in Politik und politische Institutionen sowie Eliten zwischen Ländern und über Zeiträume hinweg stark variieren (van Ham und Thomassen 2017).

Die intensive Diskussion darüber, ob das Internet Vertrauen in Politik stärken könnte, indem bürgerschaftliche Partizipation online ermöglicht wird, reicht zurück bis in die 1990er Jahre. Mehrere sogenannte Mobilisierungs-Thesen geben hierauf im Wesentlichen positive Antworten (Grossman 1995; Boulianne 2009): Die Erwartung völlig neuer Formen der Partizipation steht ebenso im Raum, wie angenommen wird, dass „,...) the Internet may reduce the costs of participation (time, effort) by increasing the availability of information“ (Boulianne 2009). Innovative (Online-)Bürgerbeteiligungsformate gelten - besonders auf lokaler Ebene - als ein mögliches Heilmittel, womit auf die „Malaisen“ der repräsentativen Demokratie zu reagieren sei (Geissel 2008; Geissel und Newton 2012).

Zwischenzeitlich finden sich in vielen Ländern und auch auf EU-Ebene eine Reihe neuer Partizipationsangebote. Allerdings belegen die Ergebnisse der empirischen Partizipationsforschung, dass politisch inaktive Bevölkerungsgruppen auch weiterhin nur schwer zu aktivieren und zu integrieren sind (vgl. Marschall und Schultze 2012). Der „democratic divide“ habe sich vielmehr sogar noch verstärkt (Brake 2008) und Online-Partizipation bleibe nach wie vor selektiv und nicht-repräsentativ (Escher 2013). Evaluationen darüber, ob mithilfe integrativer Planung oder mehr Transparenz im Prozess eine höhere Akzeptanz getroffener Entscheidungen tatsächlich zu bewirken ist, liegen aber bislang in nur begrenzter Zahl vor (Ausnahmen solcher Evaluationsversuche bieten Aichholzer und Westholm 2009; Kubicek et al. 2011).

Im vorliegenden Beitrag soll daher untersucht werden, ob innovative Online-Bürgerbeteiligungsformate überhaupt geeignet sein können, um Partizipation und letztlich das Vertrauen in staatliche Politik zu erhöhen. Denn während auf der EU-Ebene partizipative Mechanismen eher als symbolische Policy-Instrumente (Boussaguet 
2016) eingestuft werden, könnte dies in Nationalstaaten anders aussehen. Der Fokus des Beitrags liegt entsprechend nicht auf bestimmten soziologischen Gruppen und deren Integrationsfähigkeit, sondern auf der Angebotsseite der Politik. Empirisch überprüfbar sind zu diesem Zweck kommunale Plattformen auf der lokalen Ebene, die explizit mit dem Anspruch eingerichtet wurden, Online-Partizipation zu ermöglichen und Bürgerbeteiligung insgesamt zu erhöhen. Systematisch zu überprüfen sind solche Plattformen etwa auf Basis prozessorientierter oder kontextfokussierter Evaluationskriterien, wie sie von Kubicek (2014), Aichholzer und Allhutter (2008) oder Frisch (2007) vorschlagen werden.

Um die Forschungsfrage des Beitrags danach, ob Online-Partizipationsangebote die Beteiligung und letztlich das Vertrauen in staatliche Politik erhöhen können, zu beantworten, wird erstens der Forschungsstand zur Krise der Demokratie skizziert und Online-Partizipation als ein möglicher Ausweg diskutiert. Zweitens werden mit deliberativer Online-Partizipation und eVoting zwei Varianten von Liquid Democracy vorgestellt, die auf der lokalen Ebene vorzufinden sind. Sie liefern eine theoretische Begründung der Fallauswahl: Für die drittens durchgeführte Evaluation der Angebotsseite von Online-Partizipation werden sechs Fälle kommunaler Beteiligungsprojekte herangezogen, die erstens als Pioniere gelten und zweitens gleichverteilt - d.h. jeweils drei Plattformen - den beiden Liquid Democracy-Varianten zuzuordnen sind. Die sechs Plattformen werden auf Basis eines empirisch erprobten Evaluationsschemas codiert und anschließend vergleichend ausgewertet. Die Ergebnisse lassen verallgemeinerbare Aussagen darüber zu, ob und wie sich Bürgerbeteiligung über bessere Angebote erhöhen lässt. Denn es zeigt sich, dass schon die Anlagen der Plattformen einige Hürden für Bürgerbeteiligung aufweisen. Dies fußt allerdings einzig auf der Auswertung kommunaler Angebote, weil systematische Erhebungen (wie bspw. Umfragen zur Bürgerzufriedenheit oder die Kenntnis entsprechender Angebote) für die untersuchten Fälle nicht vorliegen. ${ }^{1}$ Damit findet die Studie ihre Grenzen in der Frage, welche Vorstellungen Bürger von idealer Bürgerbeteiligung haben.

\section{Zur Krise der Demokratie und (Online-)Partizipation als Ausweg?}

Die Frage nach dem Zustand der Demokratie in Deutschland lässt sich nicht pauschal beantworten. Politische Unterstützung kann von diffuser (für die Regierungsform bzw. das politische System) bis hin zu spezifischer (für eine konkrete Regierungsformation und die politische Elite) Zustimmung und Ablehnung differenziert werden (Thomassen und van Ham 2017; Norris 1999). Während die Ablehnung einer bestimmten Regierungsformation als eher unproblematisch erachtet wird, weil diese in etablierten Demokratien durch Wahlen regelmäßig ausgetauscht werden kann, wirkt die Ablehnung eines politischen Systems oder der Demokratie insgesamt ungleich

\footnotetext{
1 Dieses Vakuum kann auch die ethnografisch angelegte Vergleichs-Untersuchung von Tiemann-Kollipost (2020) nicht beheben, obwohl sie eine Ausnahme darstellt und Einblicke zu politischen Einstellungen der Nutzerinnen für die Plattform LiquidFriesland in der ursprünglichen Version enthält.
} 
schwerer. Auch erscheint es ratsam, subjektive und objektive Beurteilungen ${ }^{2}$ über den Zustand der Demokratie zu unterscheiden (Merkel und Krause 2015).

Objektiv ist demnach (im Zeitraum seit den 1970er Jahren bis heute) kein Qualitätsverlust der Demokratie in Deutschland zu beklagen (ebd., S. 53). Subjektiv wird dies etwas anders wahrgenommen: „Formale Rechte, Normen und Verfahren bleiben in der Regel intakt, aber die Chancen der Teilnahme und die tatsächliche Partizipation und Repräsentation hat sich verändert" (Merkel 2015b, S. 490). Während mehr kulturelle Sensibilität, abzulesen in der Bemühung um Gleichstellung der Geschlechter und weniger Diskriminierung ethnischer und sexueller Minderheiten, als Demokratiegewinne einzustufen ist (vgl. ebd.), hat die sozioökonomische Ungleichheit (von Vermögen, Einkommen und Lebenschancen) hingegen deutlich zugenommen (Musterd et al. 2017).

Bei genauer Betrachtung besteht demnach kein Widerspruch darin, dass die allgemeine Zufriedenheit mit repräsentativer Demokratie hoch ist (Thomassen und van Ham 2017), doch zugleich ein ,Unbehagen mit den Institutionen und den Orten [...], an denen politische Meinungen repräsentativ gebündelt und verhandelt werden“ (Bohne und Bukow 2018, S. 2) herrscht. Schließlich beruht ein solches Unbehagen auf der Unzufriedenheit der Bürger in Deutschland mit den politischen Akteuren (und daher nicht auf der diffusen, sondern der spezifischen Ebene, wodurch es nicht objektiv, sondern subjektiv ist). Einesteils sind davon Parteien betroffen, denen vorgeworfen wird, sie hätten sich von ihrer Kernklientel entfernt oder entfremdet (Merkel 2015b, S. 490). Andernteils trifft die Kritik den Regierungsstil der Bundesregierung, der als intransparent und kaum responsiv wahrgenommen wird (Pickel 2013). Die Bürger wollen also

„,nicht das politische System als solches stürzen, sondern sie sind mit ihren Vertretern unzufrieden. [...] Sie empfinden die Volksvertreter als vom Volk abgeschottet, abgehoben sowie arrogant und verlangen schlicht eine besser funktionierende Demokratie. Mittel der direkten Partizipation scheinen ihnen dabei zur Stützung der Legitimität der Demokratie am besten geeignet“ (Pickel 2013, S. 170).

Der Wunsch nach mehr bürgerschaftlichen Beteiligungsrechten, geht oftmals einher mit einem pauschalen Ruf nach mehr direkter Demokratie. Einer Bertelsmann-Studie aus dem Jahr 2014 zufolge, wünschen sich Zweidrittel der Befragten $(N=2700)$ mehr direktdemokratische Beteiligungsverfahren (vgl. Bertelsmann Stiftung 2014 „Partizipation im Wandel - Unsere Demokratie zwischen Wählen, Mitmachen und Entscheiden“). Dass überaus positive Eigenschaften, die gemeinhin den Instrumenten direkter Demokratie zugeschrieben werden - beispielsweise sachlicher, gemeinwohlorientierter und demokratischer zu sein -, auf stark verzerrten oder sogar falschen Wahrnehmungen beruhen, wird dabei allzu oft übersehen (Hornig 2016). Denn der Policy-Output von direkter Demokratie benachteiligt meist

\footnotetext{
2 Mit subjektiver Beurteilung sind Umfragewerte gemeint, die die Ansichten von Bürgerinnen und Bürgern widerspiegeln und also den ,gefühlten“ Zustand einer Demokratie aufzeigen. Objektive Beurteilungen speisen sich demgegenüber aus Demokratieindizes, die verschiedene Indikatoren heranziehen und messen oder auf Umfrageergebnissen aus Expertenbefragungen basieren.
} 
bestimmte, weniger gut organisierte Gruppen und Gesellschaftsschichten. Trotzdem sind Forderungen nach ihr - nicht nur in Deutschland, sondern EU-weit - seit einigen Jahren beständig hoch und nehmen noch weiter zu (Kriesi 2014).

Direkte Demokratie kann besonders dort ihre Vorteile entfalten, wo sie nicht als Gegenkonzept, sondern als ein komplementäres Angebot zur repräsentativen Demokratie genutzt wird. Sie kann helfen, die Akzeptanz politischer Entscheidungen zu erhöhen. Zentrale Elemente direkter Demokratie finden sich dazu bereits in verschiedenen Demokratiekonzepten (Kersting et al. 2008, S. 45 f.): In der numerischen Demokratie lassen sich etwa Abstimmungen über Sachentscheidungen als Bürgerreferenden durchführen. In der Verhandlungsdemokratie sind Bürgergruppen denkbar, um Sachpolitik miteinander auszuhandeln. In der deliberativen Demokratie kann ein offener, von gegenseitigem Respekt und Gleichheit geprägter Austausch zwischen Bürgern stattfinden. Teilweise wird dies theoretisch noch weitergedacht und mündet in modifizierten oder ganz neuen Vorschlägen, mit dem Ziel, mehr Bürgerbeteiligung im Kontext repräsentativer Demokratie zu ermöglichen (Nolte 2011; Nanz und Leggewie 2016) ${ }^{3}$.

Mit der Digitalisierung hat sich die Gemengelage partizipativer Möglichkeiten noch weiter ausdifferenziert und es sind neue Online-Angebote hinzugekommen. Nicht immer kann jedoch trennscharf zwischen Online und Offline unterschieden werden (blended democracy). Auch werden repräsentative Elemente mit deliberativen und direktdemokratischen Angeboten kombiniert eingesetzt (hybrid democracy). Schließlich ist zwischen Beteiligungsangeboten zu differenzieren, die von der Regierung initiiert sind (invited spaces) und solchen, die von den Bürgern kommen (invented spaces). Kersting (2013) hat die vorstehenden Zusammenhänge in einem komplexen Diagramm zusammengefasst (Abb. 1) und veranschaulicht damit, wie vielfältig die Angebote zur Partizipation mittlerweile sind.

Um die Angebotsseite der Politik zu beleuchten, muss es um die im Schaubild als top-down initiierten ,invited spaces“ gehen. Wenn die Frage lautet, ob die Beteiligung der Bürger durch bestimmte Angebote zur Partizipation oder deren Ausgestaltung zu erhöhen ist, so geht es nicht um repräsentative, sondern direkte oder deliberative Demokratie. Das Angebot kommunaler Plattformen wird mithilfe von Software-Tools für die Bürger zugänglich. Ein Beispiel hierfür ist das meist für direktdemokratische Abstimmungsverfahren eingesetzte Tool „LiquidFeedback“ (vgl. Abb. 1). Ein nicht im Schaubild abgetragenes Gegenstück dazu ist die Software „Adhocracy“, über die sich unter anderem deliberative Partizipation realisieren lässt. Deliberative Partizipation ist wiederum über bestimmte Verfahrensarten umzusetzen, wie etwa nicht-förmliche Konsultationen ${ }^{4}$ auf der lokalen Ebene. Welches Angebot

\footnotetext{
3 Paul Nolte spricht in seinen Ausführungen von Multipler Demokratie. Nanz, Leggewie unterbreiten den Vorschlag einer Konsultativen als bürgerschaftliches Ergänzungsgremium zum Parlament.

4 Kubicek (2014, S. 275) definiert diese folgendermaßen: „,[Nicht-förmliche Konsultationen] sind gesetzlich nicht geregelt und daher frei gestaltbar, in der Regel aber auch weniger verbindlich. Mit ihnen möchten Politik und Verwaltung in einigen Fällen ein Stimmungsbild zu bestimmten Vorhaben, in anderen inhaltliche Vorschläge zu bestimmten Fragen aufgrund des lokalen Wissens von Betroffenen erhalten. Entsprechend unterschiedlich ist die inhaltliche Offenheit der Konsultationen und auch die Verwendung der Beiträge. Thematisch wird ein breites Spektrum von Städtebau- und Raumplanungsprojekten im Rahmen der
} 


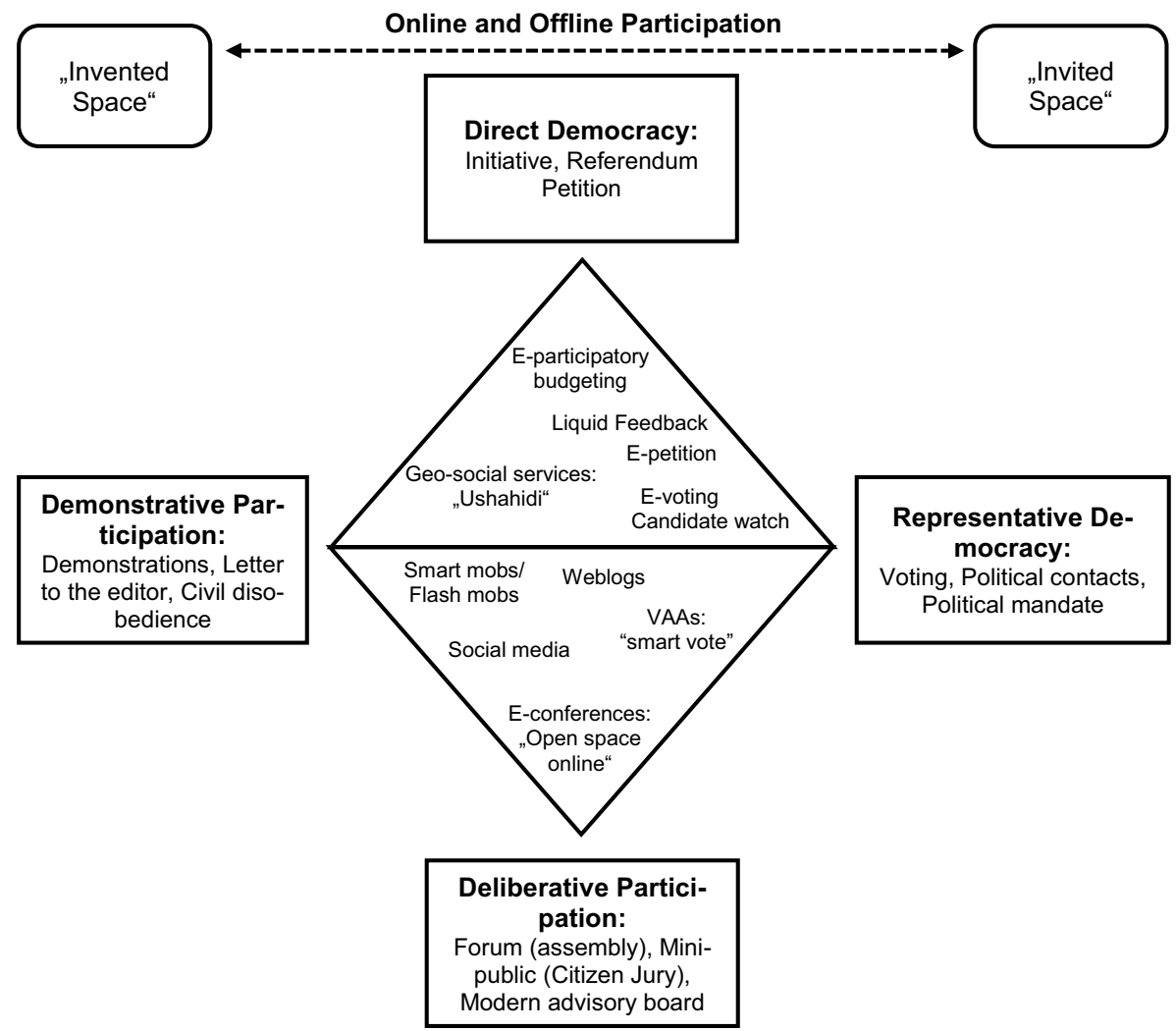

Abb. 1 Hybrid and blended democracy. (Quelle: Kersting 2013, S. 272)

sich für Online-Beteiligung besser oder schlechter eignet, ist nicht eindeutig zu beantworten.

\section{Deliberative Online-Partizipation und eVoting als Varianten von Liquid Democracy}

Die deliberative Demokratie gilt als Nachfolgetheorie der partizipatorischen Demokratietheorie und hat in der theoretischen und empirischen Auseinandersetzung viel Aufmerksamkeit erfahren (siehe zu den drei Entwicklungsphasen Schaal und Ritzi 2009, S. 7-8). Der zunehmende Einsatz in der politischen Praxis - vor allem auf kommunaler und EU-Ebene - belegt außerdem, dass deliberativen Verfahren das Potenzial zugeschrieben wird, politische Entscheidungen stärker zu legitimieren oder politische Ergebnisse qualitativ zu verbessern. In der Theorie erfüllt der Diskurs eine zentrale Funktion in der Meinungsbildung (Habermas 1981, 1994). Nach Habermas

sogenannten frühzeitigen Öffentlichkeitsbeteiligung von Gesetzesvorhaben, Leitbilddiskussionen, Haushaltsentwürfen u.a.m. abgedeckt“. 
sollen Diskurse herrschaftsfrei und konsensorientiert verlaufen, bis sich das beste Argument durchsetzt. Modi der Entscheidungsfindung folgen demnach nicht dem Mehrheitsprinzip, sondern basieren in der deliberativen Demokratie auf einem Partizipationsmodell, wonach alle möglichen gesellschaftliche Akteure miteinander im Diskurs zusammentreten. Um solche Diskurse auch online zu ermöglichen, schlägt Cohen $(1989,2007)$ klare Kriterien vor. Sie sollten auf Vernunft, einem barrierefreien Zugang, Herrschaftsfreiheit und einer guten Informationsbasis fußen. Eine erfolgreiche Deliberation fordert den Teilnehmenden einiges ab und ist grundsätzlich voraussetzungsvoll und anforderungsreich. In den Sozialwissenschaften wurde daher lange bezweifelt, ob Deliberation überhaupt eine empirische Handlungslogik für Politik und Zivilgesellschaft sein kann (Bächtiger 2015, S. 252). Kersting (2013, S. 277) kommt so auch zu dem Schluss, Deliberation sei im Online-Raum praktisch nicht umzusetzen, weil Diskurse stets nur von sehr schwacher Qualität seien: Die Habermas'schen Kriterien und Regeln würden demnach im digitalen Raum nicht angewendet und es fänden sich neben regelmäßigen respektlosen und teilweise aggressiven Foren-Beiträgen eher Serien-Monologe, die zwar individuelle Positionen und Interessen ausdrückten, aber keinen Austausch von Argumenten auf der Suche nach der besten Lösung darstellten. Dies bewirke eine „Offline-Online-Metamorphose": So werde nicht mehr diskutiert, sondern nur noch über verschiedene Optionen virtuell und direktdemokratisch abgestimmt. ,In other words, the talk centric deliberative instrument changed online into a vote-centric direct democracy instrument“ (ebd.).

Diese Einschätzung widerspricht jedoch jüngeren Entwicklungen: Mehrere deutsche Kommunen haben Online-Angebote eingerichtet, um dem Wunsch ihrer Bürger $\mathrm{zu}$ entsprechen und mehr Bürgerbeteiligung zu realisieren. Unter ihnen finden sich sowohl Möglichkeiten für Online-Abstimmungen als auch solche zur Online-Deliberation. Einige Kommunen labeln ihre Angebote dezidiert als Experimente einer Liquid Democracy (so bspw. der Landkreis Friesland).

Liquid Democracy lässt sich als ein innovatives Demokratiekonzept beschreiben, das vor rund zehn Jahren Eingang in die deutsch-sprachige Literatur gefunden hat und über zwei Varianten umzusetzen ist: Electronic Voting ${ }^{5}$ oder OnlineDeliberation $^{6}$. Liquid Democracy beruht auf einer fundamentalen Kritik an den Schwächen repräsentativer und direkter Demokratie und ist daher integrativ konstruiert, d.h. zentrale Elemente dieser Demokratiekonzepte verschmelzen in der Liquid Democracy miteinander zu einem neuen Konzept. Dem Anspruch nach soll über Online-Beteiligung Partizipation gesteigert, proportionale Repräsentation erhöht und das Vertrauen in Demokratie insgesamt gestärkt werden.

Liquid Democracy haftet der hohe (normative) Anspruch an, eine mögliche Alternative - wenn nicht sogar die bessere - zu anderen, etablierten Demokratiemodellen

\footnotetext{
5 Umgesetzt wurde diese Variante beispielsweise von der Piratenpartei, deren Bilanz insgesamt eher negativ ausfällt (Rosenfeld 2012).

6 Diese Variante wurde beispielsweise im Bundestag von der Enquete-Kommission „Internet und digitale Gesellschaft“ erprobt. Mit der Software „Adhocracy“ sollte die Öffentlichkeit in die parlamentarische Gremienarbeit eingebunden werden (https://enquetebeteiligung.de/). Eine Neuauflage der Enquete-Beteiligung lief vom 10. März bis 19. April 2020 in der Enquete-Kommission „Künstliche Intelligenz“ und über die Nachfolge-Software ,adhocracy.plus“, ist aber unter gleicher Internet-Adresse zu erreichen.
} 
zu sein (Blum und Zuber 2016). Dieser Anspruch trifft allerdings auf harsche Kritik, wonach das Konzept an zahlreichen Stellen nicht halte, was es verspreche und schon gar keine Verbesserung gegenüber bestehenden Demokratiemodellen darstelle (Dobusch und Pick 2012; Merkel 2015a): Neben der Problematik, dass aufgrund des sog. digital divide nicht alle Bürger partizipieren könnten, verschärfe Liquid Democracy mit dem Prinzip unüberschaubarer, mehrfacher Stimmendelegation und -kumulation (in der Variante von eVoting) die in der repräsentativen Demokratie ohnehin schon beklagten Mängel an Transparenz, Verantwortlichkeit und Kontrolle noch weiter.

Mit Liquid Democracy beschäftigten sich in Deutschland lange Zeit vor allem Parteienforscher (Buck 2012; Bieber und Lewitzki 2012; Adler 2013; Bullwinkel und Probst 2014; Bieber 2014; Vogelmann 2012). Ihr Interesse konzentrierte sich auf die Piratenpartei und deren rasanten Aufstieg mit beachtlichen Wahlerfolgen auf der Landesebene. Demokratietheoretiker (Dobusch und Pick 2012; Blum und Zuber 2016; Merkel 2015a; Schaal 2015) diskutieren demgegenüber die unterschiedlichen Konzeptvarianten von Liquid Democracy: Während die US-amerikanische Variante das Delegationsprinzip betont, liegt der Schwerpunkt in Deutschland auf deliberativer Demokratie und ihrer technologiegestützten Umsetzung (Bieber 2014, S. 202). Der wesentliche Unterschied der beiden Varianten besteht darin, dass Liquid Democracy im ersten Fall als Staatskonzept aufgefasst wird, während es im zweiten Fall um die Realisierung einer Beteiligungsdemokratie geht (Adler 2018, S. 150). Diese fußt auf einer netzbasierten Kommunikationspraxis, die seitens der Bürger ausgeprägte kommunikative Fähigkeiten voraussetzt (vgl. Brabanski und Kettner 2015, S. 28). Ob dies die Bürger überfordert oder die Angebote vielmehr so gestaltet sind, dass sie Partizipation stärken und Bürgerbeteiligung erhöhen, soll empirisch herausgearbeitet werden.

\section{Vergleichende Analyse von Online-Plattformen auf kommunaler Ebene}

Die Evaluation partizipativer Angebote kann politischen Akteuren und Verwaltungen helfen, ihre eigene Performanz besser einzuschätzen und zu optimieren. In der Literatur herrscht Einigkeit darüber, dass eine systematische Auswertung der Angebote unter verschiedenen Fragestellungen notwendig ist (Macintosh und Whyte 2008; Kubicek et al. 2011; Aichholzer und Allhutter 2008; Kubicek 2014). Jedoch sind Angebote zur Evaluation von (Online-)Partizipation noch immer überschaubar. Welches der empirisch erprobten oder theoretischen Schemata für die Fragestellung dieses Beitrags adaptiert und angewendet werden soll, ist im Folgenden abzuwägen.

Zielführend erscheinen nur solche Modelle, mit denen top-down installierte staatliche Online-Beteiligungsverfahren auf der lokalen Ebene zu evaluieren sind. Unbrauchbar sind demgegenüber Forschungsstrategien, die bottom-up initiierte Deliberationsverfahren untersuchen oder auf Offline-Diskurse angewendet werden (Yang 2012). Kubicek (2014) schlägt vor, den Beteiligungsprozess in fünf Phasen (Input, Aktivitäten, Output, Outcome und Impact) zu unterteilen. Mit entsprechenden Subkriterien für jede Phase und weitere prozessbegleitende Aspekte (Transparenz, 
Feedback, Effizienz, Demokratischer Einfluss) lässt sich der Gesamterfolg des Prozesses beurteilen.

Ein weiterer Vorschlag kommt von Aichholzer und Allhutter (2008). Die Autoren unterscheiden auf Grundlage der Arbeiten von Macintosh und Whyte (2008) für die Analyse von eParticipation (eConsultation, ePetitions, eDeliberation, ePolling, eVoting) drei Perspektiven. Diese sind nicht wie bei Kubicek an Prozessphasen angelehnt, sondern berücksichtigen den Beteiligungskontext. Mithilfe projektbezogener, sozioökonomischer und demokratischer Kriterien, Sub-Kriterien und dazugehörigen Indikatoren können Angebote somit mehrdimensional und kontextbezogen evaluiert werden.

Ursprünglich zur Analyse von EU-Internetforen hat Frisch (2007) ein Schema ausgearbeitet, worin sie Online-Deliberation in fünf Dimensionen (Universalität, Freiheit, Rationalität, Öffentlichkeit und Responsivität) übersetzt und damit explizit auf Cohens Anforderungen an Online-Deliberation (siehe weiter oben) eingeht. Auf Basis eines ausdifferenzierten Fragenkatalogs lassen sich die fünf Dimensionen, die sowohl Prozess-Phasen als auch -Kontexte abbilden, messen (Frisch 2007, S. 722f.). Dieser Ansatz bietet den Vorteil, dass er die beiden vorstehenden Evaluationsansätze kombiniert. Auch lässt sich die politische Angebotsseite mit diesem Katalog sinnvoll beurteilen; eine Übertragbarkeit vom EU-Kontext auf die lokale Ebene ist sinnvoll möglich und lässt sich an das Ziel der Messung anpassen.

Tab. 1 Fragenkatalog für Online-Partizipationsangebote
1. Universalität
1.1 Gibt es Barrieren beim Zugang zur Beratung?
1.2 Wie ist der Grad der Partizipation?
1.3 Verfügen alle Betroffenen über gleiche Zugangschancen?

\section{Freiheit}

2.1 Erfolgt eine Determination der Themen durch die Organisatoren der Debatte?

2.2 Ist das Verfahren frei von sowohl externen als auch internen Zwängen?

\section{Rationalität}

3.1 Stehen Einzelinteressen oder das Gemeinwohl im Vordergrund?

3.2 Erfolgt eine direkte Interaktion mit anderen Diskussionsteilnehmern?

\section{4. Öffentlichkeit}

4.1 Ist zur informierten optimalen Teilnahme der Zugang zu relevanten Informationen gewährleistet

4.2 Sind Beratungen geschlossen oder öffentlich?

4.3 Ist die Beratung offen für neue Sprecher und Themen?

\section{Responsivität}

5.1 Rechtfertigen Entscheidungsträger ihre politischen Prinzipien, Normen und Entscheidungen unter Berücksichtigung der vorgebrachten Anliegen und Kritik?

5.2 Gehen die Anliegen in den rechtsetzenden Prozess ein?

5.3 Werden politische Entscheidungsträger in den Diskussionsverlauf einbezogen?

5.4 Gibt es bei den entscheidungsrelevanten Institutionen genügend Ressourcen, um Eingaben nutzbar zu machen?

Quelle: Eigene Zusammenstellung in Anlehnung an Frisch (2007, S. 722f.) 


\subsection{Operationalisierung}

Tab. 1 weist fünf Dimensionen aus, die empirisch zu überprüfen sind. Jede Dimension - Universalität, Freiheit, Rationalität, Öffentlichkeit und Responsivität - enthält zwischen zwei und vier Fragen, die auf die Plattformangebote der Kommunen angewendet werden. Das Codebuch im Anhang (siehe Tab. 4) weist für alle Fragen die möglichen Ausprägungen und die zu vergebenden Werte aus. Die Codierungen werden damit nachvollziehbar und die Gesamtergebnisse aller Plattformen transparent und überprüfbar.

\subsection{Methodik und Daten}

Es werden sechs Fälle für die qualitative Codierung herangezogen und anschließend einem quantitativen Vergleich unterzogen. Als Fall wird in dieser Studie eine Online-Plattform definiert, die entweder auf der Software-Lösung Adhocracy (1) oder LiquidFeedback (2) basiert. Die Auswahl der Fälle umfasst Plattformen, die als Pioniere gelten, weil sie erstmals digitalen Plattformen zur kommunalen Online-Beteiligung eingerichtet haben. Indem nicht nur ein Einzelfall untersucht wird, sondern alle Plattformen einbezogen werden, für die entsprechende Daten verfügbar waren, lassen sich die Einzelergebnisse vergleichen und am Ende einige allgemeingültige Aussagen generieren.

1. Die untersuchten Plattformen OffeneKommune ${ }^{7}$, MeinBerlin.de ${ }^{8}$ und Beteiligung.in am Beispiel der Stadt Falkensee ${ }^{9}$ basieren auf dem Adhocracy-Code. Der Verein Liquid Democracy e. V. aus Berlin steht hinter der technischen Entwicklung dieser Plattformen. Die inhaltliche Initiierung und den Betrieb der Plattformen haben mit Ausnahme von OffeneKommune ${ }^{10}$ die kommunalen Verwaltungen übernommen.

2. Die zweite Software-Lösung LiquidFeedback wird vom Berliner Verein Interaktive Demokratie e. V. (vormals Public Software Group e. V.) betrieben. Dieser entwickelte in Zusammenarbeit mit der Landkreisverwaltung Friesland mit LiquidFriesland die erste kommunale Liquid Democracy-Anwendung in Deutschland, die von 2012 bis Anfang 2016 online geschaltet war. Ende Dezember startete der Landkreis Friesland eine kostengünstigere - die hier untersuchte - Version

\footnotetext{
7 Im Internet verfügbar unter: https://offenekommune.de/instance.html.

${ }^{8}$ Im Internet verfügbar unter: https://meinBerlin.de/.

9 Während die Plattform MeinBerlin weiterhin in Betrieb ist, wurde auf der Beteiligungsplattform der Stadt Falkensee mit Stand März 2020 lediglich ein Verfahren durchgeführt (zur Erstellung von Leitlinien der Bürgerbeteiligung). Die bisherige URL (https://www.beteiligung.in/falkensee) von Beteiligung.in am Beispiel der Stadt Falkensee war mit Stand Mai 2020 nicht mehr abrufbar und verweist auf eine Fehlerseite Plattform ,adhocracy+“ (https://adhocracy.plus/falkensee/). Stattdessen ist die Seite mit Stand März 2020 unter der URL https://adhocracy.plus/buro-fur-vielfalt/ abrufbar.

${ }^{10}$ Für OffeneKommune war keine einzelne Gemeinde zuständig, weshalb es sich um ein eher informelles Portal handelt. Vielmehr konnten alle interessierten Kommunen (über NamederKommune.offeneKommune.de) die Plattform nutzen.

${ }^{11}$ Im Internet verfügbar unter: https://www.liquidfriesland.de/.
} 
der Plattform ${ }^{11}$, die seither auf der technischen Infrastruktur Bürgertipps der Nolis $\mathrm{GmbH}^{12}$ basiert. Auf der ursprünglichen Version von Liquid Friesland basieren die Bürgerplattform ROW ${ }^{13}$ des Landkreises Rotenburg (Wümme) und SeelzeDirekt ${ }^{14}$ der niedersächsischen Stadt Seelze, die ebenfalls untersucht werden ${ }^{15}$.

Die Codierungen und Re-Codierungen der Plattformen wurden von Januar bis März 2019, im Mai 2019 sowie von Februar bis März 2020 durchgeführt.

Der Datenzugang wurde von der Zugänglichkeit der untersuchten Plattformen bestimmt: Drei der untersuchten Plattformen waren zum Zeitpunkt der Datenerhebung und Codierung entweder noch (Beteiligung.in/falkensee, MeinBerlin) oder nach zwischenzeitlicher Abschaltung wieder (LiquidFriesland) online verfügbar. Drei weitere Plattformen sind mittlerweile vom Netz gegangen (Bürgerplattform ROW, SeelzeDirekt) oder nicht mehr zur Nutzung freigeschaltet (OffeneKommune). Für die Erhebung der Daten wurden daher, wenn möglich, die Plattformen Online untersucht oder alternativ auf das Internet-Archiv Wayback Machine ${ }^{16}$ zurückgegriffen.

Die Plattformen und ihre Internetseiten bzw. die Offline verfügbaren Dokumente der Plattformen werden inhaltsanalytisch mithilfe der in Tab. 1 dargestellten Fragen und anhand des ausgearbeiteten Codebuchs (siehe Tab. 4 im Anhang) ausgewertet. Durch das Codebuch sind alle Codierungs-Entscheidungen nachvollziehbar. Auf Basis des Codier-Schemas wird jeder Frage ein abgestufter Wertebereich $(-2,-1,0$, 1,2) zugeordnet und dieser auf die Fälle angewendet. Je nachdem, ob eine Frage eindeutig positiv $(+2)$ oder negativ $(-2)$ zu beantworten ist oder Einschränkungen bzw. Uneindeutigkeit vorliegt $(-1,0,1)$, wird der entsprechende Wert vergeben.

Eine Null dokumentiert Uneindeutigkeit in Bezug auf die Beantwortung der Frage oder aber, dass die Frage nicht beantwortet werden kann (kein Wert=0). Das Codebuch enthält eine Liste aller Ausprägungen und die jeweils zugewiesenen Werte.

Nach der Codierung, die auf den im Codebuch beschriebenen qualitativen Maßstäben beruht, werden die Werte aufsummiert und ergeben ein für die entsprechende Dimension nummerisches Gesamtergebnis (Index). In der Addition der Werte aller Dimensionen ergibt sich nach diesem Codier-Schema ein maximal zu erzielender Wert von 28 Punkten $^{17}$ pro Fall. Für die sechs Fälle kann sich über die fünf Di-

\footnotetext{
12 Im Internet verfügbar unter: https://www.nolis.de/portal/seiten/buergertipps-900000023-10006.html, https://www.buergertipps.de/. Eine wesentliche Änderung im Vergleich zur Original-Version besteht darin, dass eingereichte Vorschläge nicht mehr kommentiert, sondern nur noch von der Landkreisverwaltung beantwortet werden.

13 Ursprünglicher Link: www.buergerplattformrow.de (Webseite nicht mehr aktiv).

14 Ursprünglicher Link: www.SeelzeDirekt.de (Webseite nicht mehr aktiv).

15 Nicht untersucht wird aufgrund mangelnder empirischer Daten die Plattform Wunstorf-Direkt der niedersächsischen Stadt Wunstorf, die ebenfalls kurze Zeit nach LiquidFriesland ans Netz gegangen war. Ursprünglicher Link: www.wunstorf-direkt.de (Webseite nicht mehr aktiv).

16 https://archive.org/web/.

17 Dieser Wert teilt sich in folgende Maximalpunkte pro Dimension (pro Fall) auf: 6 Punkte für Universalität, 4 Punkte für Freiheit, 4 Punkte für Rationalität, 6 Punkte für Öffentlichkeit und 8 Punkte für Responsivität.
} 
mensionen hinweg also ein maximaler Gesamtwert von 168 Punkten ergeben ${ }^{18}$. Da die Anzahl der Fragen über Dimensionen hinweg variieren, werden für die bessere Vergleichbarkeit außerdem Prozentwerte ermittelt. So lassen sich zusätzlich zu den Kommunen auch die Dimensionen miteinander vergleichen.

\subsection{Evaluation kommunaler Plattformen}

Die Evaluation der sechs Fälle, gemessen über fünf Dimensionen hinweg, hat einige interessante Befunde ergeben, die mithilfe von vier Schwerpunkten dargestellt werden sollen: Erstens wird das Gesamtergebnis eingeordnet und bewertet. Zweitens sind die Dimensionen differenziert zu betrachten. Für jede Dimension wird dazu aufgezeigt, wie gut bzw. schlecht diese im Vergleich zu den anderen abgeschnitten hat. Drittens werden die Ergebnisse der Plattformen dargestellt und hinsichtlich der verwendeten Software strukturiert und verglichen. Viertens werden die - über alles betrachtet - besten und schlechtesten Einzelwerte noch einmal herausgehoben und im Kontext ihrer jeweiligen Plattform erläutert und interpretiert.

In Tab. 2 sind die codierten, aufsummierten Werte aller Plattformen und Dimensionen abgetragen (vgl. Tab. 3 mit Einzelwerten je Frage). Ihr Gesamtwert liegt bei 56 Punkten. Dieser beträgt ein Drittel der maximal zu erzielenden 168 Punkte. Insgesamt zeigt dies, dass die sechs untersuchten Plattformen ihre Angebote zur Online-Partizipation zum Zeitpunkt der Erhebung unterdurchschnittlich ausgestaltet haben. Allerdings zeigt Tab. 2 auch deutliche Unterschiede sowohl zwischen den Dimensionen als auch den Plattformen. Eine differenzierte Betrachtung und genaue Auswertung ist daher für die weitere Interpretation nötig.

Differenzierter wird die Einschätzung, wenn man die fünf Dimensionen einzeln betrachtet: Es fällt auf, dass die Dimension Universalität als einzige einen negativen Gesamtwert aufweist und damit insgesamt am schlechtesten abschneidet. Alle anderen Dimensionen erzielen positive Werte; die Dimensionen Öffentlichkeit und Responsivität liegen unter ihnen mit je $56 \%$ auf den Spitzenplätzen (vgl. Abb. 2). Rationalität liegt mit $46 \%$ an dritter Stelle.

Tab. 2 Codierungsergebnisse der kommunalen Plattformen

\begin{tabular}{llllllll}
\hline $\begin{array}{l}\text { Dimensionen/ } \\
\text { Plattformen }\end{array}$ & $\begin{array}{l}\text { Offene- } \\
\text { Kom- } \\
\text { mune }\end{array}$ & $\begin{array}{l}\text { Mein- } \\
\text { Berlin }\end{array}$ & $\begin{array}{l}\text { Beteili- } \\
\text { gung.in/ } \\
\text { Falkensee }\end{array}$ & $\begin{array}{l}\text { Liquid- } \\
\text { Fries- } \\
\text { land }\end{array}$ & $\begin{array}{l}\text { Bürgerplatt- } \\
\text { form } \\
\text { ROW }\end{array}$ & $\begin{array}{l}\text { Seelze- } \\
\text { Direkt }\end{array}$ & Summe \\
\hline Universalität & 0 & 3 & 0 & -2 & -6 & -5 & -10 \\
Freiheit & 4 & -2 & 0 & 0 & 3 & 3 & 8 \\
Rationalität & 2 & 3 & 4 & -1 & 2 & 1 & 11 \\
Öffentlichkeit & 4 & 3 & 4 & 3 & 3 & 3 & 20 \\
Responsivität & 3 & 6 & 6 & 6 & 3 & 3 & 27 \\
Gesamt & 13 & 13 & 14 & 6 & 5 & 5 & 56 \\
\hline
\end{tabular}

Quelle: eigene Zusammenstellung

18 Dieser Wert teilt sich in folgende Maximalpunkte (alle Fälle aufaddiert) pro Dimension: 36 Punkte für Universalität, 24 Punkte für Freiheit, 24 Punkte für Rationalität, 36 Punkte für Öffentlichkeit und 48 Punkte für Responsivität. 


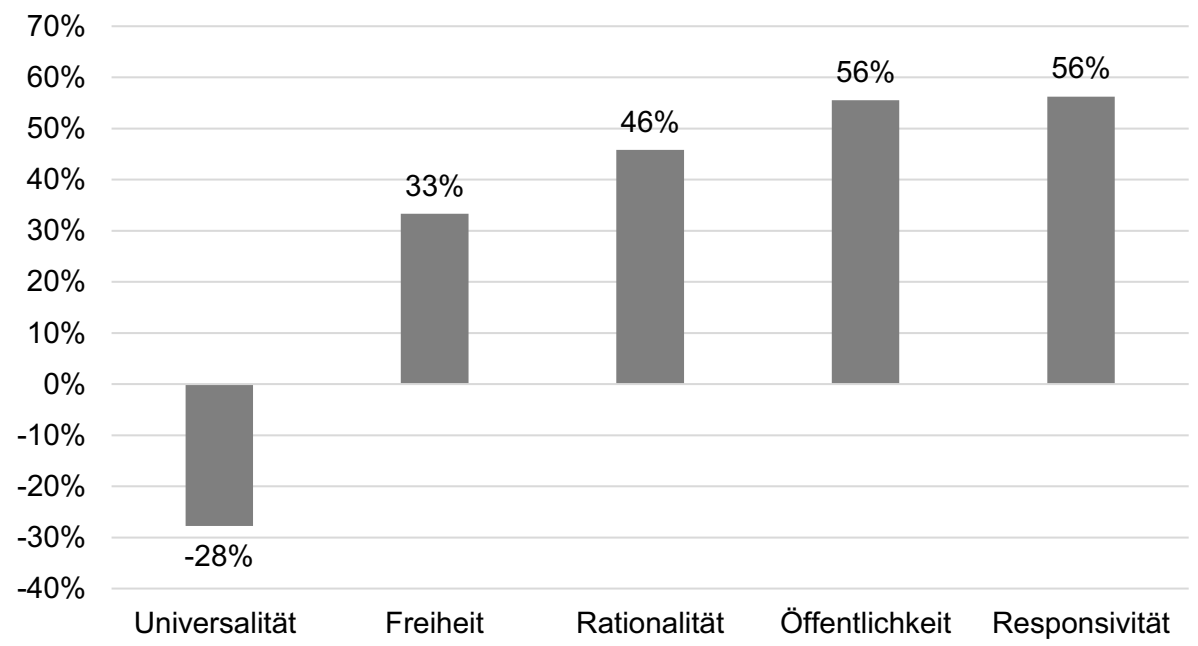

Abb. 2 Ergebnisse nach Dimensionen (in Prozent). (Quelle: eigene Darstellung)

Was sagen diese Werte aus? Dass Universalität besonders schlecht umgesetzt wird (nur eine Plattform, nämlich MeinBerlin, erhält in dieser Dimension positive Werte, vgl. Tab. 2 und 3) zeigt, dass sich den Bürgern in einem frühen Stadium des Beteiligungsprozesses, und zwar beim (technischen) Zugang zur Plattform, bereits einige Hürden stellen. Fast alle Kommunen erschweren demnach Online-Partizipation schon zu Beginn des Prozesses.

Demgegenüber wird im Verlauf des Beteiligungsprozesses vorbildlich mit den Eingaben der Bürger umgegangen: Hohe Werte für Responsivität und Öffentlichkeit (und nur mit einer negativen Ausnahme im Falle von LiquidFriesland auch für Rationalität, vgl. Tab. 2) zeigen erstens, dass eine Rückkopplung der politischen Repräsentanten an die eingebrachten Wünsche und Interessen der Bürger (bzw. der sich auf der Plattform beteiligenden Nutzer) gut realisiert wird (Responsivität). Zweitens wird auch eine Begleitung des politischen Diskurses durch eine kritische Öffentlichkeit gewährleistet, indem Informationen unterwegs zugänglich sind und eine Beobachtung des Diskurses von außen möglich ist (Öffentlichkeit). Drittens sind die Plattformen so aufgebaut, dass Interaktionen zwischen Nutzern möglich ist und auch Einzelinteressen berücksichtigt werden (können) (Rationalität).

Als nächstes soll der Blick auf die sechs kommunalen Plattformen gelegt werden. Abb. 3 ermöglicht dazu einen prozentualen Vergleich. 100\% und damit die volle Punktzahl von 28 erzielt keine Plattform. An der Spitze steht Beteiligung.in/ Falkensee mit 50\% und einem absoluten Wert von 14 Punkten. Den zweithöchsten Wert erzielen die Plattformen OffeneKommune und MeinBerlin. Sie sind mit $46 \%$ und jeweils 13 Punkten nur knapp, nämlich vier Prozentpunkte hinter dem Spitzenreiter. Die drei weiteren Plattformen LiquidFriesland, SeelzeDirekt sowie die Bürgerplattform ROW folgen mit deutlichem Abstand auf den nächsten Plätzen und liegen mit Werten von 21 und $18 \%$ dicht beieinander (vgl. Abb. 3).

Das Ranking der kommunalen Beteiligungsangebote zeigt ein deutliches Muster: Plattformen, die auf der Software Adhocracy basieren, dominieren das Feld 


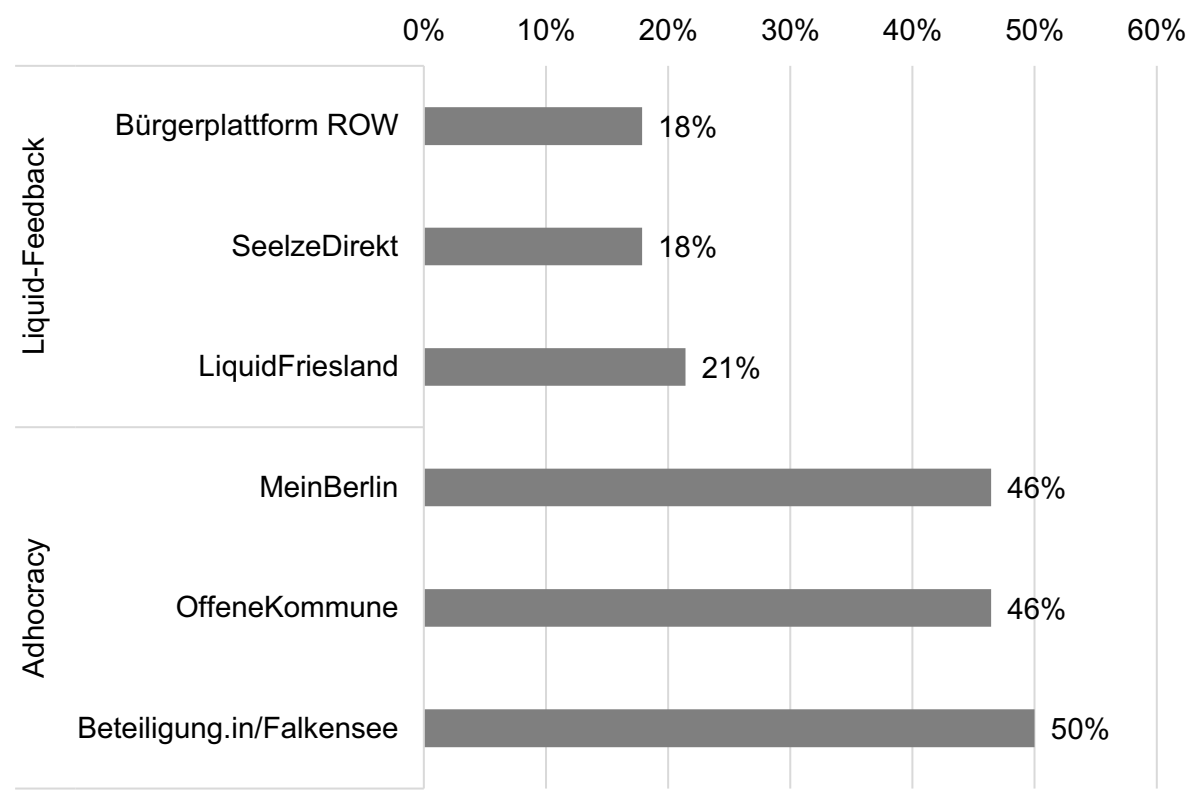

Abb. 3 Ergebnisse nach Plattformen (in Prozent). (Quelle: eigene Darstellung)

gegenüber Plattformen, die mit LiquidFeedback arbeiten. Die Angebotsseite wird demnach qualitativ von der Wahl der Software vorstrukturiert; Adhocracy schneidet im Vergleich besser ab als LiquidFeedback-Angebote.

Weniger relevant scheint es hingegen für die Platzierung zu sein, ob die Plattformen nach einer Pilotphase wieder vom Netz genommen wurden (OffeneKommune, SeelzeDirekt, Bürgerplattform ROW, Beteiligung.in/falkensee), weiterhin online sind (MeinBerlin) oder nach kurzzeitiger Abschaltung und Neuauflage erneut online geschaltet worden sind (LiquidFriesland).

Die bisherigen Ergebnisse lassen sich grafisch mithilfe von farbigen Netzdiagrammen komplex abbilden und vergleichen. Im Fünfeck der untersuchten Dimensionen (vgl. Abb. 4) wird sichtbar, welche kommunalen Plattformen entlang der untersuchten Dimensionen die besten und die schlechtesten Einzelwerte erzielen konnten. Augenfällig ist hierbei, dass drei Kommunen (MeinBerlin, Beteiligung.in/Falkensee und LiquidFriesland) hohe Punktwerte bei Responsivität erzielen konnten. Die Bürgerplattform ROW erzielt bei Universalität den insgesamt schlechtesten Wert.

Wie ist dies zu erklären? Die hohen Werte bei Responsivität sind auf drei zentrale Aspekte zurückzuführen: Erstens werden Transparenzregeln in allen drei Kommunen angewendet. Dies betrifft den Umgang mit den Ergebnissen. Bürger können also nachvollziehen, was mit ihren Eingaben passiert und wie ihre eingebrachten Wünsche entschieden worden sind. Was aus ihnen wird - auch nach Ende des offiziellen Beteiligungsprozesses -, ist somit für alle Akteure nachvollziehbar. Zweitens werden politische Entscheider in den Online-Beteiligungsprozess einbezogen, d.h. sie können sich selbst daran beteiligen und zu einzelnen Vorgängen, Wünschen und Eingaben Stellung nehmen. Schließlich tätigen die Kommunen drittens vergleichsweise 


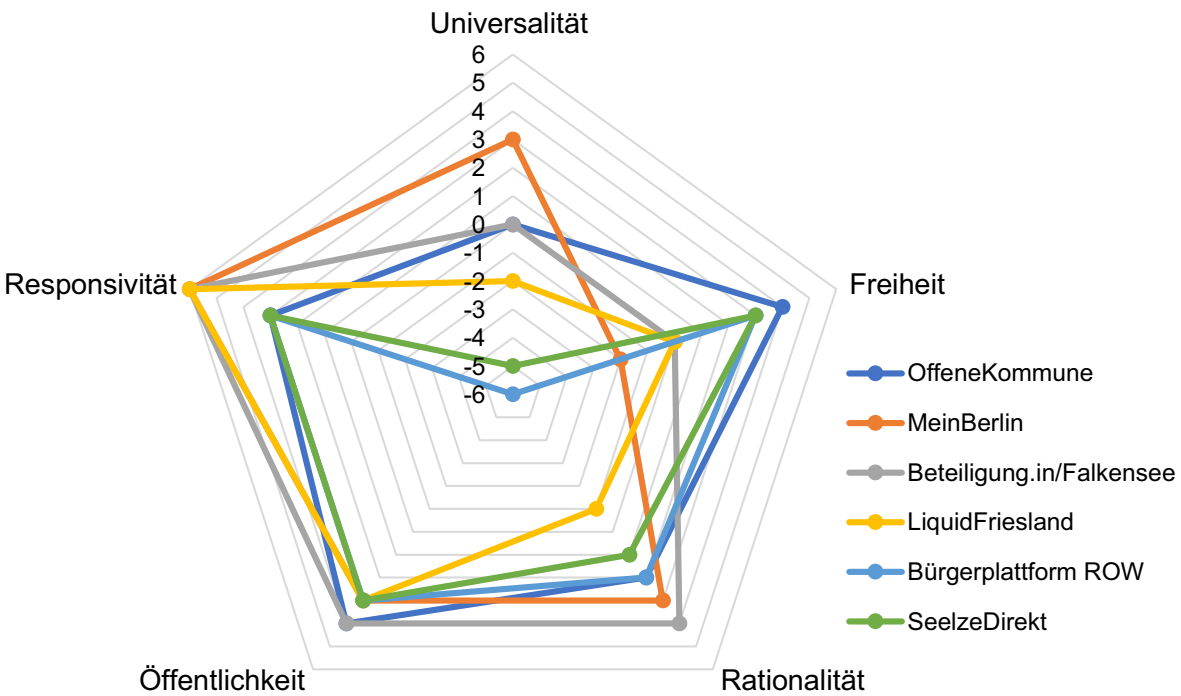

Abb. 4 Netzdiagramme für sechs kommunale Plattformen. (Quelle: eigene Darstellung)

hohe investive Maßnahmen, um die Plattformen zu betreiben. Diese Maßnahmen umfassen sowohl den Einsatz personeller als auch materieller Ressourcen.

Der schlechteste Wert wurde in der Dimension Universalität erzielt. Gründe hierfür liegen in der deutlichen Einschränkung beim Zugang zur Plattform: So herrscht erstens eine Pflicht zur Registrierung bei fast allen Plattformen, was das insgesamt schlechte Abschneiden der Dimension erklärt. Zweitens können sich bei der Hälfte der Plattformen (Bürgerplattform ROW, Liquid-Friesland und Seelze-Direkt) nur Nutzer beteiligen, die einen lokalen Bezug zur Kommune haben und diesen auch durch ihren Erstwohnsitz nachweisen können. Eine solche Einschränkung wiegt schwer, weil sie Ausschlüsse produziert, die zu geringerer Online-Beteiligung auf der Plattform führen (können).

Die (zu) geringe Beteiligung wird als ein generelles Problem von (Online-)Partizipation angesehen. Einerseits liegt das an den Bürgern, wenn diese nicht teilnehmen. Andererseits liegt die Verantwortung bei der Verwaltung, wenn Ausschlüsse oftmals sogar aktiv verursacht werden, anstatt diese zu verhindern. Gründe für die Nicht-Beteiligung der Bürger lassen sich vielfach im Internet finden. Denn hier wird die öffentliche Sphäre aufgelöst, an der alle aktiv oder passiv (beispielsweise massenmedial vermittelt als Zuschauer) teilnehmen können. Im Netz finden sog. (Selbst-)Selektionseffekte statt, die Deliberation hemmen (vgl. Schaal 2015, S. 298; Schaal und Ritzi 2009). Hinzu kommt, dass Menschen eher in virtuelle Räume streben, wo sie ihre Meinungen und Ansichten bestätigt finden. Die zielgruppenspezifische Ausdifferenzierung von Medienangeboten lädt demnach nicht dazu ein, sich in anstrengende und konträre Diskussionen zu begeben, mit dem Ziel, in InternetForen die beste politische Lösung für ein spezifisches Policy-Problem auszuhandeln. Staat und Verwaltung müssen darum aktiv daran mitarbeiten ,ein breites Interesse 
an Foren zur Erörterung politischer Entscheidungen [zu] wecken“ (Albrecht et al. 2008, S. 137 f.), wenn sie entsprechende Angebote unterbreiten.

Die Ausgestaltung der Angebote sind somit von essenzieller Bedeutung. Ein zentrales Ergebnis der Studie ist, dass die Angebote in den sechs Kommunen - über fünf Dimensionen hinweg gemessen - sehr unterschiedlich ausgestaltet sind und sich entlang der Software strukturieren lassen. Besonders im frühen Stadium des Beteiligungsprozesses wurden Defizite in fast allen Kommunen festgestellt, während im Verlauf und gegen Ende gute Werte erzielt werden konnten.

Andere, vor dem Beteiligungsprozess liegende, Faktoren wurden in die Analyse nicht einbezogen. Für sie zeigt sich erstens eine sehr gute Breitbandversorgung und Netzabdeckung als Grundvoraussetzung für Online-Beteiligung in den jeweiligen Kommunen. Die Versorgungwerte liegen in allen sechs Kommunen mit 50 Mbit/s im bzw. deutlich über dem Bundesdurchschnitt von 91,9\% aller Haushalte. ${ }^{19}$

$\mathrm{Ob}$ und wie allerdings zweitens von Seiten der Kommunen für die Angebote im Vorfeld der Beteiligungsverfahren geworben worden ist, kann aufgrund fehlender Dokumentationen nur vorsichtig beurteilt werden. So lassen etwa die Bürgerplattform ROW und SeelzeDirekt erkennen, dass ihre Öffentlichkeitsarbeit zu Beginn Informationsveranstaltungen, Flyer und Pressemitteilungen umfasste, die dann aber weder systematisch noch kontinuierlich fortgeführt wurde. ${ }^{20}$ Dabei liegt genau hier ein regelmäßig auftretendes zentrales Problem:

,[Es] wird immer wieder auf Marketingmaßnahmen zur Bekanntmachung von Beteiligungsangeboten verzichtet, weil man hofft, eine Pressemitteilung und eigendynamische Diffusionsprozesse im Internet würden ausreichen. Dies ist ein Irrtum, der fast immer mit einer geringen Teilnehmerzahl quittiert wird. Erst recht werden in diesen Fällen diejenigen Bevölkerungsgruppen außen vor gelassen, die bereits in verschiedener Hinsicht als unterprivilegiert einzustufen sind. Für solche Personengruppen, z. B. Migranten und Personen mit niedrigeren Bildungsabschlüssen, muss in der Regel eine Zielgruppen-genaue Ansprache gewählt werden“ (Albrecht et al. 2008, S. 137 f.).

So ist schließlich drittens zu bedenken, dass sich Bildungsunterschiede, sozioökonomische Unterschiede und Sprachbarrieren in der Bevölkerung negativ-selektiv auf Beteiligung auswirken (können). Umgekehrt können Beteiligungstools und -verfahren in ,leichter Sprache“, so belegen es jüngste empirische Studien, Partizipation erhöhen (vgl. Fink et al. 2020).

19 Mit 50 Mbit/s (in \% der Haushalte, Datenstand Ende 2019) versorgt sind die Stadt Seelze (99\%), die Stadt Berlin (98\%), die Stadt Falkensee (95\%), der Landkreis Rotenburg (Wümme) (90\%) und der Landkreis Friesland (88\%). Quelle: Breitbandatlas des Bundes, (C BMVI, atene KOM.

20 Mitteilungsvorlage der Stabstelle Kreisentwicklung vom 24.02.2017, Drucksachen-Nr.: 2016-21/0131: https://sessionnet.lk-rotenburg.de/sessionnet/bi/getfile.php?id=44608\&type=do\&;

Niederschrift der 2. Sitzung des Ausschusses für Personal- und Organisationsentwicklung am 07.03.2017 des Landkreises Rotenburg (Wümme): https://sessionnet.lk-rotenburg.de/sessionnet/bi/getfile.php?id=45093\& type $=$ do\&;

Informationsvorlage Nummer: XVI/0663a vom 05.02.2016 der Stadt Seelze: https://seelze.morerubin1.de/show_pdf.php?_typ_432=vor1\&_doc_n1=73009101391.pdf\&_nk_nr=7\&_nid_nr=73009101391\&_ neu_dok=\&status=1\&sitzungsnummer=ni_2016-3-79. 
Abschließend soll der Aspekt der (Verfahrens-)Transparenz noch einmal aufgegriffen und reflektiert werden. Wenn etwa Schneider (2018, S. 75) konstatiert, innovative Beteiligungsverfahren simulierten lediglich die Abgabe von Macht, Responsivität und mehr Beteiligung, weil etablierte Machtstrukturen und Entscheidungsprozesse weiterhin unverändert bestehen blieben, so bewegt diese Aussage sicherlich nicht zu mehr Bürgerbeteiligung. Auch wird damit unterstellt, eine direkte Mitwirkung von Bürgern legitimiere Politikentscheidungen nur scheinbar, aber nicht tatsächlich besser. Dieser Einschätzung ist $\mathrm{zu}$ widersprechen: Denn die politische Berücksichtigung der Belange der Bürger, die sich einbringen, mindestens eine Befassung damit, ist hochgradig mitbestimmend dafür, ob sich Bürger ganz grundsätzlich, wieder und auch weiterhin politisch beteiligen. Und dies transparent zu kommunizieren ist genauso wichtig. Umgekehrt kann Intransparenz im Beteiligungsprozess schlimmstenfalls das Gegenteil bewirken und Inaktivität noch befördern. Denn die Mehrzahl der Bürger wünscht sich transparente Beteiligungsprozesse (vgl. Bauser 2019).

\section{Zusammenfassung und Fazit}

Im Zentrum des Beitrags stand die Frage, ob innovative Online-Bürgerbeteiligungsformate dafür geeignet sein können, Partizipation zu erhöhen.

Ausgangspunkt war die Beobachtung zweier paralleler Entwicklungen: Einerseits durchzieht die digitale Transformation die Gesellschaft in allen denkbaren Lebensbereichen und bringt vielfältige neue Chancen und Gefahren mit sich. Andererseits hat die Zufriedenheit mit Demokratie bzw. konkret das Vertrauen in politische Institutionen, Prozesse und Akteure, in den letzten Jahren abgenommen. Dabei ist die Literatur keineswegs einhellig in der Frage, ob wir es mit einer Krise der Demokratie zu tun haben oder nicht.

Aus der Verbindung dieser parallelen Entwicklungen wurde die Frage gestellt, ob die Digitalisierung etwas dazu beitragen kann, das Vertrauen in die Demokratie zu stärken. Mit mehreren Mobilisierungsthesen wurde dies in den 1990er Jahre zunächst positiv beantwortet. Die Erwartung war, dass völlig neue Partizipationsformen entstünden und Online-Beteiligung einfacher und vielfältiger werden würde. Dieser Optimismus wurde schon bald gedämpft: Empirische Studien zeigen auf, dass Partizipation auch in Zeiten des Internets selektiv bleibt und sich der sog. democratic divide eher noch verschärft. Es beteiligen sich demnach Online wie Offline weiterhin nur wenige, aktive Menschen. Evaluationen zu dahinter liegenden Gründen und der Frage, ob mehr Transparenz tatsächlich eine bessere Akzeptanz für politische Entscheidungen bewirken kann, liegen noch immer nicht zahlreich vor.

Sind die eingesetzten Policy-Instrumente und Angebote zur Partizipation auf der lokalen Ebene förderlich im Sinne der Partizipation oder bleiben sie rein symbolisch, wie es über eine lange Zeit für die EU-Ebene konstatiert wurde?

Diese Frage ist mithilfe der vorgelegten Studie zu beantworten. Die Angebote zur Partizipation sind mittlerweile zahlreich - darunter analoge, digitale und blended; angesiedelt auf verschiedenen gebietskörperschaftlichen Ebenen (Kommune, Land, Bund, EU); initiiert von Seiten des Staates oder von Bürgern (vgl. Kersting 2013). 
Untersucht wurden lokale Plattformen, die als Angebote direkter und deliberativer Demokratie einzustufen sind, daraufhin, ob die Angebote zu mehr Partizipation anregen oder nicht. Dafür wurden sechs Plattformen, die empirisch als Pioniere gelten, auf Basis eines empirisch erprobten und für diese Studie stark abgewandelten Analyseschemas qualitativ codiert, quantitativ ausgewertet und anschließend miteinander verglichen.

Im Ergebnis hat sich gezeigt, dass die Plattformen unterschiedlich ausgestaltet sind und sich entlang der verwendeten Software klar strukturieren lassen. Plattformen, die Adhocracy verwenden, schneiden demnach besser ab als Plattformen, die auf LiquidFeedback basieren. Differenziert nach Dimensionen hat sich außerdem gezeigt, dass Universalität vergleichsweise schlecht umgesetzt worden ist. Damit ist der Zugang zum Beteiligungsprozess in einer frühen Phase des Verfahrens mit Hürden versehen, was eine Beteiligung schon zu Beginn hemmen kann. Anders zeigen sich die Dimensionen Öffentlichkeit und Responsivität - also die Informationen nach innen und außen sowie Transparenz im politischen Prozess: all dies wurde gut umgesetzt, befinden sich aber im Verlauf bzw. am Ende des Beteiligungsprozesses und kann mitentscheidend dafür sein, ob sich der aktive Bürger auch weiterhin beteiligt oder dies künftig unterlässt. Systematische Erhebungen darüber, warum sich Bürger individuell (nicht) beteiligen, liegen für die untersuchten Fälle nicht vor, weshalb sich die Studie auf die Auswertung der Angebotsseite beschränken musste. Anekdotische Rückmeldungen von Nutzern sowie Protokollausschnitte von Ratssitzungen deuten jedoch eine Bestätigung der zentralen Ergebnisse dieser Studie an (siehe dazu verschiedene Dokumente, wie Ratsdokumente von Rotenburg/Wümme ${ }^{21}$, ein Ratsprotokoll von Friesland ${ }^{22}$, die Medienberichterstattung zu Seelze $^{23}$ oder eine Bürgerbeteiligungsstudie für Falkensee ${ }^{24}$ ). Demnach müssten die Hürden für eine Beteiligung rigoros gesenkt werden, während gleichzeitig die Verwaltung ihren Einsatz zur Betreuung der Angebote intensivieren sollte. Nur so ließe sich dem - entgegen aller optimistischen Mobilisierungsthesen - anhaltend negativen Trend einer geringen Partizipation entgegenwirken.

Es lässt sich damit festhalten, dass es einerseits höchst voraussetzungsvoll ist, Online-Beteiligungsverfahren so zu gestalten, dass Bürger - unabhängig von sozioökonomischen Unterschieden und individuellen Technikkenntnissen - aktiv mitmachen. Es erfordert andererseits seitens der Kommunalverwaltung einen großen

\footnotetext{
${ }^{21}$ Mitteilungsvorlage der Stabstelle Kreisentwicklung vom 24.02.2017, Drucksachen-Nr.: 2016-21/0131: https://sessionnet.lk-rotenburg.de/sessionnet/bi/getfile.php?id=44608\&type=do\&;

Niederschrift der 2. Sitzung des Ausschusses für Personal- und Organisationsentwicklung am 07.03.2017 des Landkreises Rotenburg (Wümme): https://sessionnet.lk-rotenburg.de/sessionnet/bi/getfile.php?id=45093\& type $=$ do\&.

22 Sitzung des Kreistag des Landkreises Friesland am 25.06.2013, TOP Ö 6.4.5: Fortführung von LiquidFriesland: https://buergerinfo.friesland.de/to0050.asp?_ktonr=9337.

${ }^{23}$ Tschörner, Thomas (2016): Stadt will Online-Beteiligung einstellen, Neue Presse, 30.09.2016, https:// www.neuepresse.de/Region/Seelze/Nachrichten/Seelze-stellt-die-Online-Buergerbeteiligung-Seelzedirektein.

${ }^{24}$ AgoraKomm \& arf Gesellschaft für Organisationsentwicklung mbH (2017): Durchführung einer Studie zum Konzept der Bürgerbeteiligung in der Stadt Falkensee. Studienergebnisse zum Status Quo, 12.04.2017, https://daten2.verwaltungsportal.de/dateien/seitengenerator/170407_falkensee_studie_druck. pdf.
} 
personellen, zeitlichen und auch finanziellen Einsatz, wenn Angebote für OnlinePartizipation einen Nutzen für die Bürger haben und nicht von vornherein nur Schaufenstermaßnahmen sein sollen.

Funding Open Access funding provided by Projekt DEAL.

Open Access Dieser Artikel wird unter der Creative Commons Namensnennung 4.0 International Lizenz veröffentlicht, welche die Nutzung, Vervielfältigung, Bearbeitung, Verbreitung und Wiedergabe in jeglichem Medium und Format erlaubt, sofern Sie den/die ursprünglichen Autor(en) und die Quelle ordnungsgemäß nennen, einen Link zur Creative Commons Lizenz beifügen und angeben, ob Änderungen vorgenommen wurden.

Die in diesem Artikel enthaltenen Bilder und sonstiges Drittmaterial unterliegen ebenfalls der genannten Creative Commons Lizenz, sofern sich aus der Abbildungslegende nichts anderes ergibt. Sofern das betreffende Material nicht unter der genannten Creative Commons Lizenz steht und die betreffende Handlung nicht nach gesetzlichen Vorschriften erlaubt ist, ist für die oben aufgeführten Weiterverwendungen des Materials die Einwilligung des jeweiligen Rechteinhabers einzuholen.

Weitere Details zur Lizenz entnehmen Sie bitte der Lizenzinformation auf http://creativecommons.org/ licenses/by/4.0/deed.de. 


\section{Anhang}

Tab. 3 Ergebnistabelle mit kodierten Einzelwerten

\begin{tabular}{|c|c|c|c|c|c|c|c|}
\hline \multirow{2}{*}{\multicolumn{2}{|c|}{$\begin{array}{l}\text { Software } \\
\text { Plattform }\end{array}$}} & \multicolumn{3}{|c|}{ Adhocracy } & \multicolumn{3}{|c|}{ Liquid-Feedback } \\
\hline & & \multirow{2}{*}{$\begin{array}{l}\text { Offene- } \\
\text { Kom- } \\
\text { mune } \\
-1\end{array}$} & \multirow{2}{*}{$\begin{array}{l}\text { Mein- } \\
\text { Berlin } \\
-1\end{array}$} & \multirow{2}{*}{$\begin{array}{l}\text { Beteili- } \\
\text { gung.in/ } \\
\text { Falken- } \\
\text { see } \\
-1\end{array}$} & \multirow{2}{*}{$\begin{array}{l}\text { Liquid- } \\
\text { Fries- } \\
\text { land } \\
1\end{array}$} & \multirow{2}{*}{$\begin{array}{l}\text { Bürger- } \\
\text { plattform } \\
\text { ROW } \\
-2\end{array}$} & \multirow{2}{*}{$\begin{array}{l}\text { Seelze- } \\
\text { Direkt } \\
\\
-2\end{array}$} \\
\hline $\begin{array}{l}\text { Univer- } \\
\text { salität }\end{array}$ & $\begin{array}{l}\text { 1.1 Gibt es Barrieren beim } \\
\text { Zugang zur Beratung? }\end{array}$ & & & & & & \\
\hline & $\begin{array}{l}1.2 \text { Ist das Forum für alle } \\
\text { individuellen Beteiligungs- } \\
\text { wünsche offen? }\end{array}$ & -1 & 2 & -1 & -1 & -2 & -1 \\
\hline & $\begin{array}{l}\text { 1.3 Wie ist der Grad der Parti- } \\
\text { zipation? }\end{array}$ & 2 & 2 & 2 & -2 & -2 & -2 \\
\hline \multirow[t]{2}{*}{ Freiheit } & $\begin{array}{l}2.1 \text { Ist das Verfahren frei von } \\
\text { sowohl externen als auch } \\
\text { internen Zwängen? }\end{array}$ & 2 & -2 & 0 & 2 & 2 & 2 \\
\hline & $\begin{array}{l}2.2 \text { Erfolgt eine Determina- } \\
\text { tion der Themen durch die } \\
\text { Organisatoren der Debatte? }\end{array}$ & 2 & 0 & 0 & -2 & 1 & 1 \\
\hline \multirow[t]{2}{*}{$\begin{array}{l}\text { Ratio- } \\
\text { nalität }\end{array}$} & $\begin{array}{l}\text { 3.1 Stehen Einzelinteressen } \\
\text { oder das Gemeinwohl im } \\
\text { Vordergrund? }\end{array}$ & 0 & 2 & 2 & 1 & 0 & 0 \\
\hline & $\begin{array}{l}\text { 3.2 Erfolgt eine direkte Inter- } \\
\text { aktion mit anderen Diskussi- } \\
\text { onsteilnehmern? }\end{array}$ & 2 & 1 & 2 & -2 & 2 & 1 \\
\hline \multirow[t]{3}{*}{$\begin{array}{l}\text { Öffent- } \\
\text { lichkeit }\end{array}$} & $\begin{array}{l}\text { 4.1 Ist zur informierten Teil- } \\
\text { nahme der Zugang zu relevan- } \\
\text { ten Informationen gewährleis- } \\
\text { tet? }\end{array}$ & 0 & 2 & 2 & 0 & 0 & 0 \\
\hline & $\begin{array}{l}\text { 4.2 Sind Beratungen geschlos- } \\
\text { sen oder öffentlich? }\end{array}$ & 2 & -1 & 2 & 2 & 1 & 1 \\
\hline & $\begin{array}{l}4.3 \text { Ist die Beratung offen für } \\
\text { neue Sprecher und Themen? }\end{array}$ & 2 & 2 & 0 & 1 & 2 & 2 \\
\hline \multirow[t]{4}{*}{$\begin{array}{l}\text { Respon- } \\
\text { sivität }\end{array}$} & $\begin{array}{l}\text { 5.1 Rechtfertigen Entschei- } \\
\text { dungsträger ihre politischen } \\
\text { Prinzipien, Normen und Ent- } \\
\text { scheidungen unter Berück- } \\
\text { sichtigung der vorgebrachten } \\
\text { Anliegen und Kritik? }\end{array}$ & 2 & 2 & 2 & 2 & 2 & 2 \\
\hline & $\begin{array}{l}5.2 \text { Gehen die Anliegen in den } \\
\text { rechtsetzenden Prozess ein? }\end{array}$ & -1 & 1 & 1 & 1 & -1 & -1 \\
\hline & $\begin{array}{l}\text { 5.3 Werden politische Ent- } \\
\text { scheidungsträger in den Dis- } \\
\text { kussionsverlauf einbezogen? }\end{array}$ & 2 & 1 & 1 & 1 & 0 & 0 \\
\hline & $\begin{array}{l}\text { 5.4 Gibt es bei den entschei- } \\
\text { dungsrelevanten Institutionen } \\
\text { genügend Ressourcen, um } \\
\text { Eingaben nutzbar zu machen? }\end{array}$ & 0 & 2 & 2 & 2 & 2 & 2 \\
\hline
\end{tabular}

Quelle: Eigene Darstellung. 


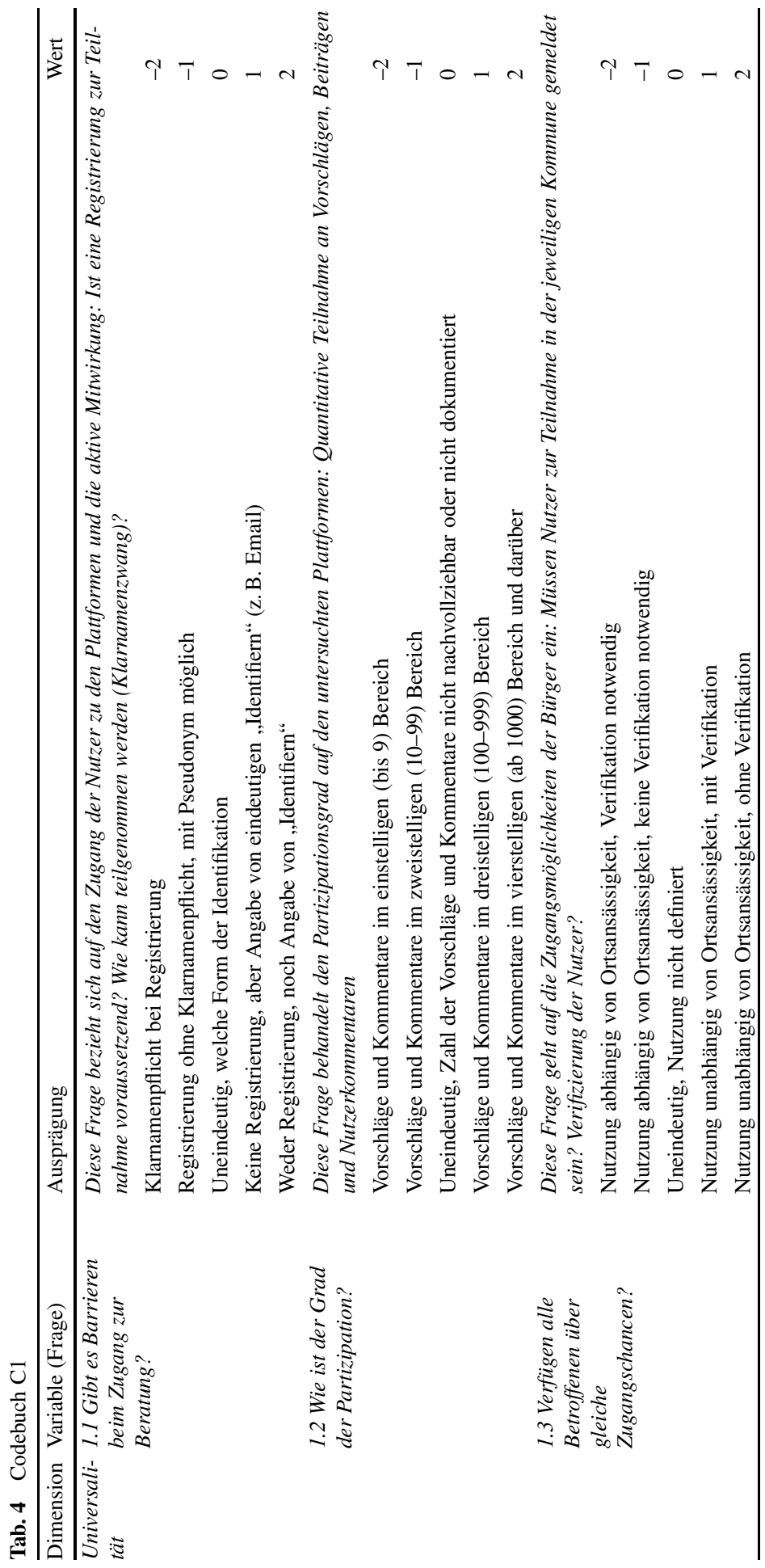




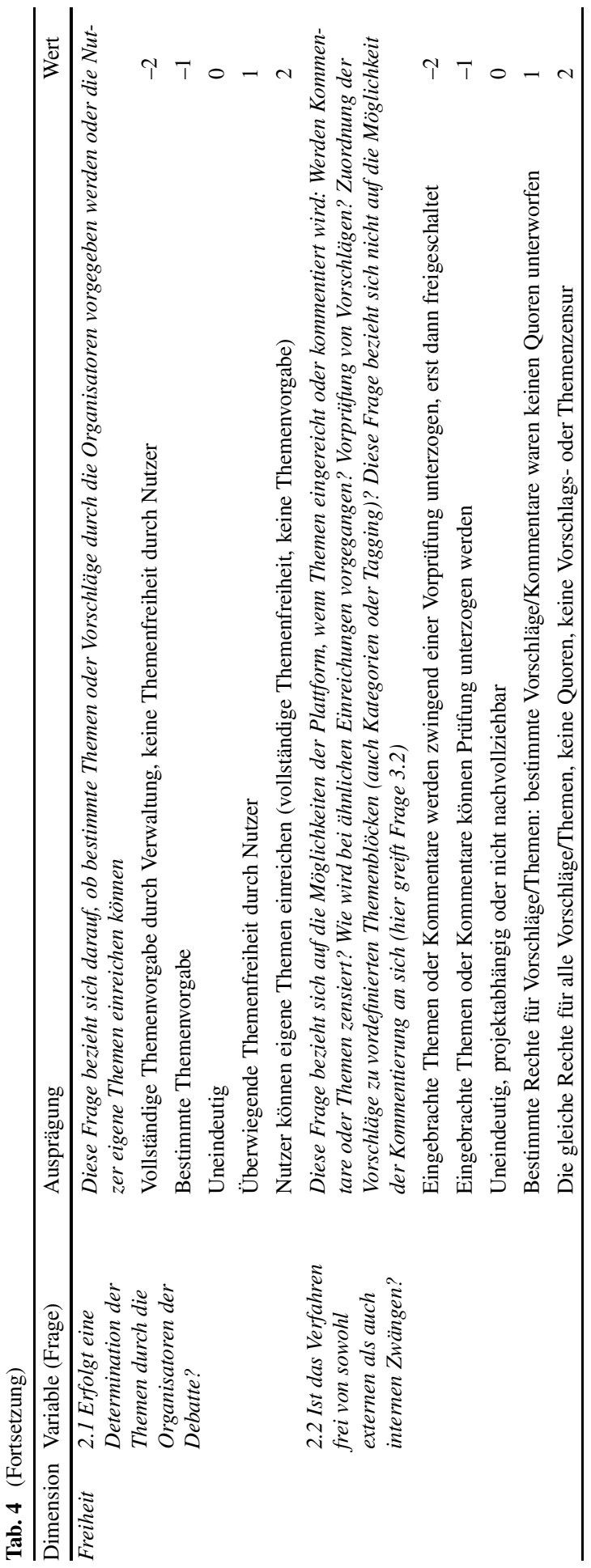




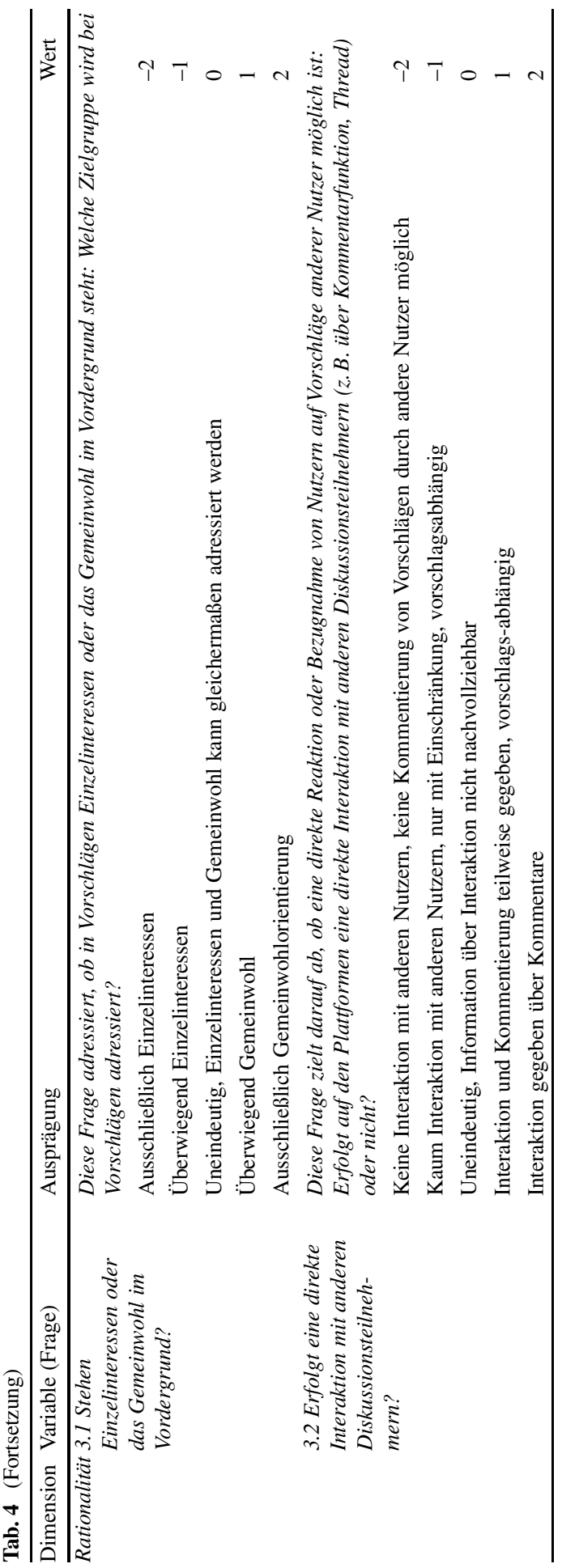




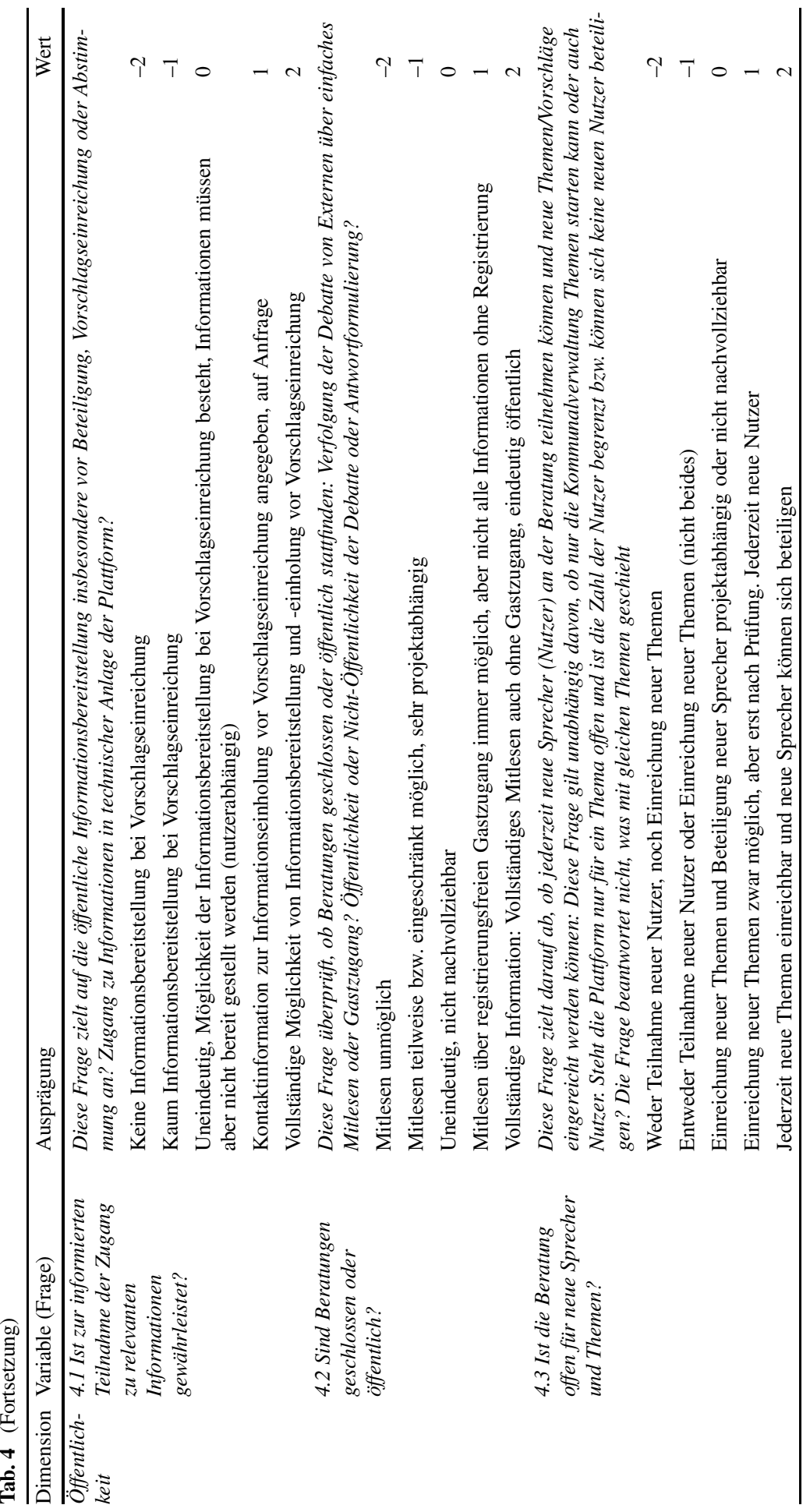




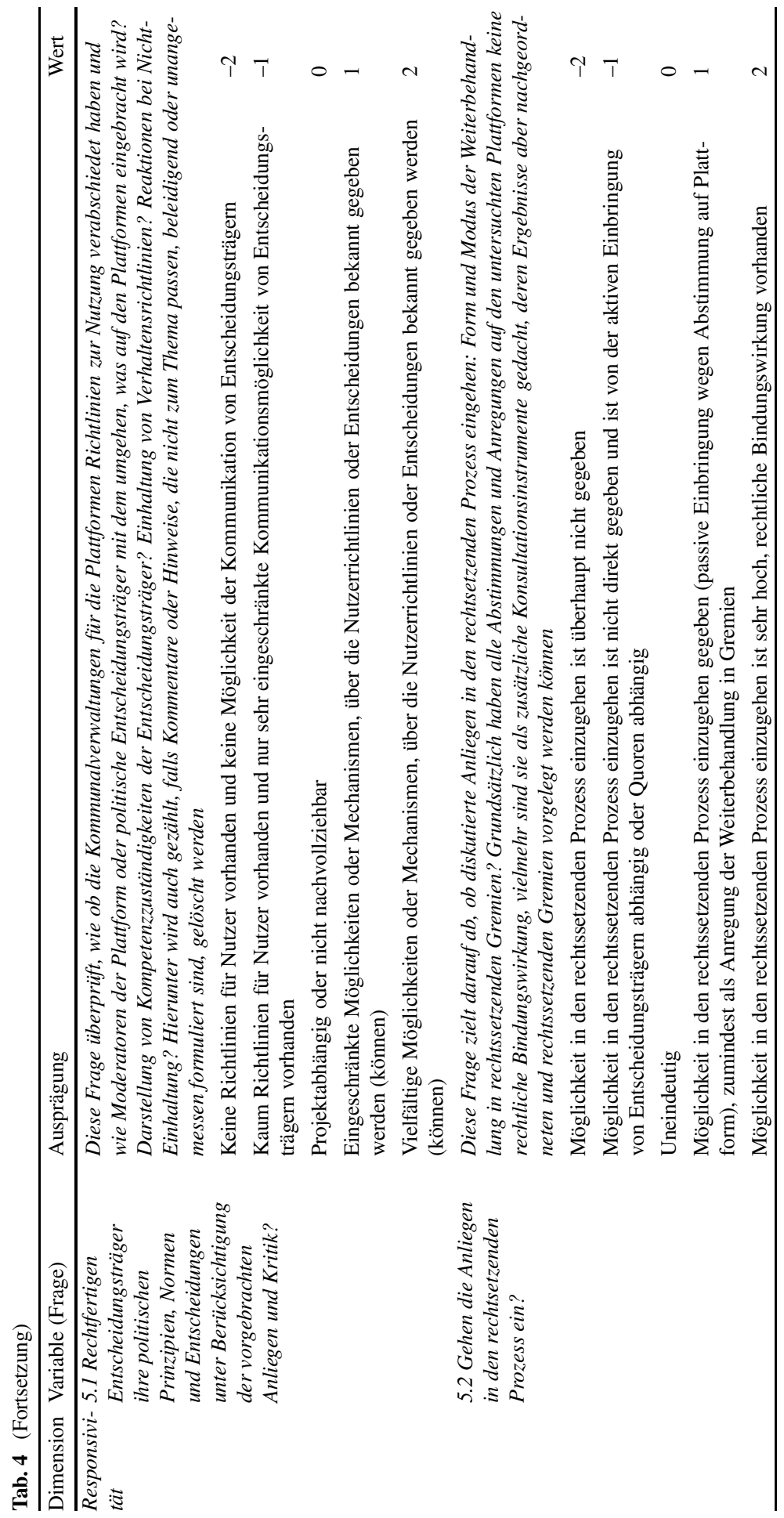




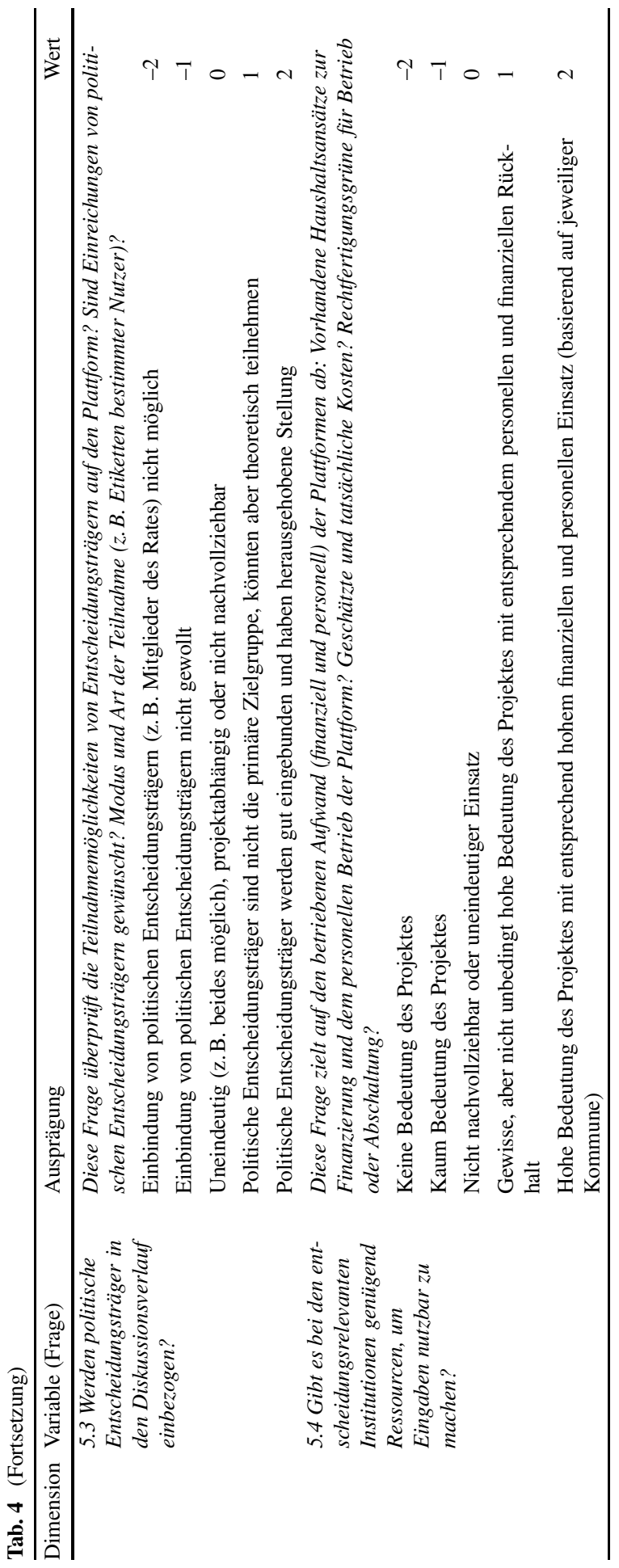




\section{Literatur}

Adler, Anja. 2013. Liquid Democracy als Social Software für Parteien. Forschungsjournal Soziale Bewegungen 26(2):71-83.

Adler, Anja. 2018. Liquid Democracy in Deutschland. Zur Zukunft digitaler politischer Entscheidungsfindung nach dem Niedergang der Piratenpartei. Bd. 59. Bielefeld: transcript.

Aichholzer, G., und H. Westholm. 2009. Evaluating eparticipation projects. Practical examples and outline of an evaluation framework. European Journal of ePractice 7:27-44.

Aichholzer, Georg, und Doris Allhutter. 2008. Evaluation Perspectives and Key Criteria in eParticipation. https://s3.amazonaws.com/academia.edu.documents/33074043/GA_DA_eeegovdays2008. pdf?response-content-disposition=inline \%3B\%20filename\%3DEvaluation_Perspectives_and_Key_ Criteria.pdf\&X-Amz-Algorithm=AWS4-HMAC-SHA256\&X-Amz-Credential=AKIAIWOWYYG Z2Y53UL3A\%2F20200320\%2Fus-east-1\%2Fs3\%2Faws4_request\&X-Amz-Date=20200320T1319 $18 Z \& X-A m z-E x p i r e s=3600 \& X-A m z-S i g n e d H e a d e r s=$ host $\& X-A m z-S i g n a t u r e=812 \mathrm{ffda} 39 \mathrm{cfdd} 569 \mathrm{e}$ 9b81ea909d6438938af69a5d06eee3712a3667530f27a6b. Zugegriffen: 16.06.2020.

Albrecht, Steffen, Niels Kohlrausch, Herbert Kubicek, Barbara Lippa, Oliver Märker, Matthias Trénel, Volker Vorwerk, Hilmar Westholm, und Christian Wiedwald. 2008. E-Partizipation - Elektronische Beteiligung von Bevölkerung und Wirtschaft am E-Government. Studie im Auftrag des Bundesministeriums des Innern. Ref. ITI

Bächtiger, André. 2015. Empirische Deliberationsforschung. In Empirische Demokratietheorien Zeitgenössische Demokratietheorie, Bd. 2, Hrsg. Oliver W. Lembcke, Gary Schaal, und Claudia Ritzi, 252-278. Wiesbaden: Springer VS.

Bauser, Johannes, 2019. Erfolgsbedingungen für Bürgerbeteiligung. Einfluss kommunaler Entscheidungsträger auf Beteiligungsergebnisse am Beispiel Liquid Feedback. Unveröffentlichte Dissertation.

Bertelsmann Stiftung. 2014. Vielfältige Demokratie. Kernergebnisse der Studie „Partizipation im Wandel - Unsere Demokratie zwischen Wählen, Mitmachen und Entscheiden “. Studie im Auftrag des Staatsministeriums Baden-Württemberg.

Bieber, Christoph. 2014. Innovation und Politische Beteiligung. Von der Computer-Demokratie zur Liquid Democracy. In Handbuch Innovationen, Hrsg. Manfred Mai, 189-207. Wiesbaden: Springer VS.

Bieber, Christoph, und Markus Lewitzki. 2012. Die Piratenpartei. Organisieren ohne Organisation? In Wie entscheiden Parteien?, Hrsg. Karl-Rudolf Korte, Jan Treibel, 219-248. Baden-Baden: Nomos.

Blum, Christian, und Christina Isabel Zuber. 2016. Liquid democracy: potentials, problems, and perspectives. The Journal of Political Philosophy 24(2):162-182.

Bohne, Maik, und Sebastian Bukow. 2018. Selbstbewusstsein statt Unbehagen. Impulse zur Stärkung der repräsentativen Demokratie in Deutschland. Policy Brief/Discussion Paper. Berlin: Das Progressive Zentrum.

Boulianne, Shelley. 2009. Does Internet use affect engagement? A meta-analysis of research. Political Communication 26(2):193-211.

Boussaguet, Laurie .2016. Participatory mechanisms as symbolic policy instruments? Comparative European Politics 14:107-124. https://doi.org/10.1057/cep.2015.12.

Brabanski, Oskar, und Matthias Kettner. 2015. Chancen und Risiken von Liquid Democracy für die politische Kommunikation. In Cyber-Sicherheit. Studien zur Inneren Sicherheit, Bd. 18, Hrsg. H.J. Lange, A. Bötticher, 19-35. Wiesbaden: Springer VS.

Brake, Anne. 2008. Internetbasierte Befragung - ein Instrument für den Weg in eine aktive Bürgersellschaft? In Politische Beteiligung: Einführung in dialogorientierte Instrumente politischer und gesellschaftlicher Partizipation, Bd. 28, Hrsg. Norbert Kersting, 65-79. Wiesbaden: Springer VS.

Buck, Sebastian. 2012. Liquid Democracy - Eine Realisierung Deliberativer Hoffnungen? Zum Selbstverständnis der Piratenpartei. Zeitschrift Für Parlamentsfragen 43(3):626-635.

Bullwinkel, Bastian, und Lothar Probst. 2014. Innerparteiliche Willensbildung und Entscheidungsprozesse durch digitale Partizipation. Ein Praxistest des Konzepts der Liquid Democracy. Zeitschrift für Parlamentsfragen 45(2):382-401.

Cohen, Joshua. 1989. Deliberation and democratic legitimacy. In The Good Polity: Normative Analysis of the State, Hrsg. Alan Hamlin, Phillip Petit, 17-34. New York: Blackwell.

Cohen, Joshua. 2007. Deliberative democracy. In Deliberation, participation and democracy, Hrsg. S.W. Rosenberg, 219-236. London: Palgrave Macmillan.

Dobusch, Leonard, und Yussi Pick. 2012. Parteidemokratie \#4: Liquid Democracy in Theorie und Praxis [Update], blog.sektionacht.at. http://blog.sektionacht.at/2012/06/parteidemokratie-4-liquiddemocracy-in-theorie-und-praxis/. Zugegriffen: 30. Sept. 2018. 
Escher, Tobias. 2013. Mobilisierung zu politischer Partizipation durch das Internet: Erwartungen, Erkenntnisse und Herausforderungen der Forschung. Analyse \& Kritik 35(2):449-476.

Fink, Simon, Eva Ruffing, Tobias Burst, und Katharina Chinnow. 2020. Less complex language, more participation. How consultation documents shape participatory patterns. Working Paper.

Frisch, Annika. 2007. Das Potenzial deliberativer Demokratietheorie für die Konzeptionalisierung von Demokratie in der Europäischen Union. Zeitschrift für Politikwissenschaft 17(3):711-733.

Geissel, Brigitte. 2008. Zur Evaluation demokratischer Innovationen - die lokale Ebene. In Lokale Politikforschung heute, Hrsg. Hubert Heinelt, Angelika Vetter, 227-248. Wiesbaden: Springer VS.

Geissel, Brigitte, und Kenneth Newton (Hrsg.). 2012. Evaluating democratic innovations. London, New York: Routledge.

Grossman, Lawrence K. 1995. The electronic republic. Reshaping democracy in the information age. New York: Viking.

Habermas, Jürgen. 1981. Theorie des kommunikativen Handelns. Bd. 1, 2. Frankfurt a.M.: Suhrkamp.

Habermas, Jürgen. 1994. Faktizität und Geltung, 4. Aufl., Frankfurt a.M.: Suhrkamp.

Hornig, Eike-Christian. 2016. Zu viel Rousseau, zu wenig Fraenkel. Zur Debatte über direkte Demokratie in Deutschland. In Politik mit Bürgern - Politik für Bürger, Bürgergesellschaft und Demokratie, Hrsg. Manuela Glaab, 273-288. Wiesbaden: Springer VS.

Kerstin, Norbert, Philippe Schmitter, und Alexander Trechsel. 2008. Die Zukunft der Demokratie. In Politische Beteiligung: Einführung in dialogorientierte Instrumente politischer und gesellschaftlicher Partizipation, Bd. 28, Hrsg. Norbert Kersting, 40-62. Wiesbaden: Springer VS.

Kersting, Norbert. 2008. Politische Beteiligung: Einführung in dialogorientierte Instrumente politischer und gesellschaftlicher Partizipation. Bd. 28. Wiesbaden: Springer VS.

Kersting, Norbert. 2013. Online participation: From invited to invented spaces. Int. J. Electronic Governance 6(4):270-280.

Kriesi, Hanspeter. 2013. Democratic legitimacy: Is there a legitimacy crisis in contemporary politics? Politische Vierteljahresschrift 54(4):609-638.

Kriesi, Hanspeter. 2014. Was die Europäer unter Demokratie verstehen und wie sie die Demokratie in ihrem eigenen Land bewerten. In Demokratie, Diktatur, Gerechtigkeit, Hrsg. Aurel Croissant, Sascha Kneip, und Alexander Petring, 353-370. Wiesbaden: Springer VS.

Kubicek, Herbert. 2014. Staatliche Beteiligungsangebote im Internet - ein Überblick. In Internet und Partizipation. Bottom-up oder Top-down? Politische Beteiligungsmöglichkeiten im Internet, Hrsg. Kathrin Voss, 265-298. Wiesbaden: Springer VS.

Kubicek, Herbert, B. Lippa, und A. Koop. 2011. Erfolgreich beteiligt? Nutzen und Erfolgsfaktoren internet-gestützter Bürgerbeteiligung - Eine empirische Analyse von zwölf Fallbeispielen. Gütersloh: Bertelsmann Stiftung.

Macintosh, A., und A. Whyte. 2008. Towards an evaluation framework for eparticipation. Transforming Government: People, Process \& Policy 2(1):16-30.

Marschall, Stefan, und Martin Schultze. 2012. Voting advice applications and their effect on voter turnout: the case of the German Wahl-O-mat. International Journal of Electronic Governance 5:3-4.

Merkel, Wolfgang. 2015a. Nur schöner Schein? Demokratische Innovationen in Theorie und Praxis. OBSArbeitsheft 80:5-23.

Merkel, Wolfgang. 2015b. Schluss. Ist die Krise der Demokratie eine Erfindung? In Demokratie und Krise, Hrsg. Wolfgang Merkel, 473-498. Wiesbaden: Springer VS.

Merkel, Wolfang, und Werner Krause. 2015. Krise der Demokratie? Ansichten von Experten und Bürgern. In Demokratie und Krise, Hrsg. Wolfgang Merkel, 45-66. Wiesbaden: Springer VS.

Musterd, Sako, Szymon Marcińczak, Maarten van Ham, und Tiit Tammaru. 2017. Socioeconomic segregation in European capital cities. Increasing separation between poor and rich. Urban Geography 38(7):1062-1083.

Nanz, Patrizia, und Claus Leggewie. 2016. Die Konsultative. Mehr Demokratie durch Bürgerbeteiligung. Berlin: Verlag Klaus Wagenbach.

Nolte, Paul. 2011. Von der repräsentativen zur multiplen Demokratie. APuZ 61(1-2):5-12.

Norris, Pippa. 1999. Critical citizens: global support for democratic governance. Oxford: Oxford University Press.

Offe, Claus. 2003. Demokratisierung der Demokratie. Diagnosen und Reformvorschläge. Frankfurt a.M.: Campus Verlag.

Pähle, Katja. 2008. Bürgerbeteiligung auf kommunaler Ebene. Eine Herausforderung für die Legitimation lokaler Mandatsträger? In Lokale Politikforschung heute, Hrsg. Hubert Heinelt, Angelika Vetter, 249-269. Wiesbaden: Springer VS. 
Pickel, Susanne. 2013. Politische Kultur, Systemvertrauen und Demokratiezufriedenheit. Wann fühlen sich die Bürger gut regiert? In Handbuch Regierungsforschung, Hrsg. Karl-Rudolf Korte, Timo Grunden, 161-174. Wiesbaden: Springer VS.

Rosenfeld, Dagmar, 2012: Wenn alle mit allen über alles reden. Immer, in: DIE ZEIT Nr. 18/2012.

Schaal, Gary. 2015. E-Democracy. In Empirische Demokratietheorien Zeitgenössische Demokratietheorie, Bd. 2, Hrsg. Oliver W. Lembcke, Gary Schaal, und Claudia Ritzi, 279-306. Wiesbaden: Springer VS.

Schaal, Gary, und Claudia Ritzi. 2009. Empirische Deliberationsforschung. MPIfG Working Paper 09/9.

Schneider, Sebastian. 2018. Bürgerhaushalte in Deutschland. Individuelle und kontextuelle Einflussfaktoren der Beteiligung. Wiesbaden: Springer VS.

Thomassen, Jacques, und Carolien van Ham. 2017. A legitimacy crisis of representative democracy? In Myth and reality of the legitimacy crisis: explaining trends and cross-national differences in established democracies, Hrsg. Carolien van Ham, Jacques Thomassen, Kees Aarts, und Rudy Andeweg. Oxford: Oxford University Press.

Tiemann-Kollipost, Julia. 2020. Political participation in the digital age: an ethnographic comparison between Iceland and Germany. Bielefeld: transcript.

van Ham, Carolien, und Jacques Thomassen. 2017. The myth of legitimacy decline: an empirical evaluation of trends on political Suppert in Estabilshed democracies. In Myth and reality of the legitimacy crisis: explaining trends and cross-national differences in established democracies, Hrsg. Carolien van Ham, Jacques Thomassen, Kees Aarts, und Rudy Andeweg. Oxford: Oxford University Press.

van Ham, Carolien, Jacques Thomassen, Kees Aarts, und Rudy Andeweg. 2017. Myth and reality of the legitimacy crisis: explaining trends and cross-national differences in established democracies. Oxford: Oxford University Press.

Vogelmann, Frieder. 2012. Flüssige Betriebssysteme. Liquid Democracy als demokratische Machttechnologie. ApuZ 48:40-46.

Yang, Mundo. 2012. Deliberative Politik von unten. Eine diskursanalytische Feldstudie dreier politischer Kleingruppen. Baden-Baden: Nomos.

Zittel, Thomas. 2012. Wie viel und welche Partizipation braucht die Demokratie? vorgänge 3:4-14. 